\title{
ON A QUASILINEAR DEGENERATE SYSTEM ARISING IN SEMICONDUCTORS THEORY. PART I: EXISTENCE AND UNIQUENESS OF SOLUTIONS*
}

\author{
J.I. DíAZ ${ }^{\dagger}$, G. GALIANO ${ }^{\ddagger}$, AND ANSGAR JÜNGEL §
}

\begin{abstract}
A drift-diffusion model for semiconductors with nonlinear diffusion is considered. The model consists of two quasilinear degenerate parabolic equations for carrier densities and the Poisson equation for electric potential. We assume Lipschitz continuous nonlinearities in the drift and generation-recombination terms.

Existence of weak solutions is proven by using a regularization technique. Uniqueness of solutions is proven when either the diffusion term $\varphi$ is strictly increasing and solutions have spatial derivatives in $L^{1}\left(Q_{T}\right)$ or when $\varphi$ is non-decreasing and a suitable entropy condition is fullfilled by the electric potential.
\end{abstract}

Key words. Quasilinear degenerate system, semiconductors.

AMS subject classifications. 35K65, 35D05, 35B30, 78A35.

1. Introduction. In solid state physics, drift-diffusion equations are today the most widely used model to describe semiconductor devices. Drift-diffusion models describe the flow of electrons and holes in the conduction and valence band, respectively, of semiconductor materials which are influenced by an electric field. Mathematically, the problem is formulated in terms of a system of parabolic equations for the electron and hole densities $u, v$, and the Poisson equation for the electric potential $w$ which together with physically motivated auxiliary conditions form the problem

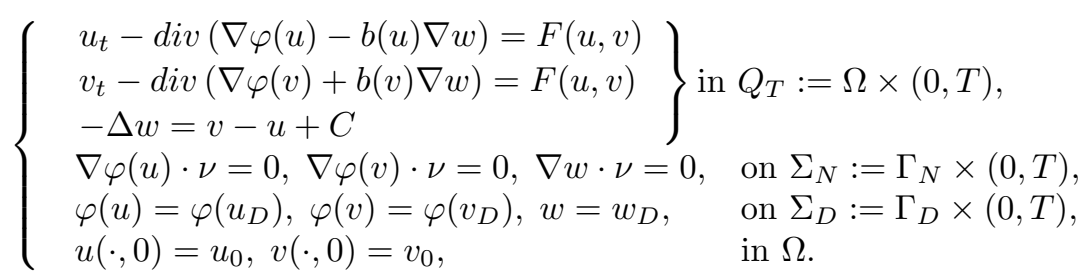

with $\Omega \subset \mathbb{R}^{N}, 1 \leq N \leq 3$, the bounded domain occupied by the semiconductor crystal. Here, function $C$ denotes the doping profile (fixed charged background ions) characterizing the semiconductor under consideration, $\varphi$ the pressure function, $b(s) / s$ the mobility of the particles, and $F$ the recombination-generation rate. The boundary $\partial \Omega$ splits into two disjoint subsets $\Gamma_{D}$ and $\Gamma_{N}$. Carrier densities and potential are fixed at the Ohmic contacts, $\Gamma_{D}$, whereas their fluxes are null on $\Gamma_{N}$, the union of insulating boundary segments.

The standard drift-diffusion model corresponds to linear functions $\varphi$ and $b$, and $F(u, v)=q(u, v)\left(u_{i}^{2}-u v\right)$, with $q(u, v)$ a positive function and $u_{i} \equiv u_{i}(x)>0$ the so-called intrinsic density. The standard model can be derived from Boltzmann's

\footnotetext{
${ }^{\dagger}$ Departamento de Matemática Aplicada, Universidad Complutense de Madrid, 28040 Madrid, Spain (jidiaz@sunma4.mat.ucm.es). Partially sponsored by the DGICYT (Spain) project PB96/0385.

¥Departamento de Matemáticas, Universidad de Oviedo, 33007 Oviedo, Spain (galiano@orion.ciencias.uniovi.es).

$\S$ Fachbereich Mathematik, Universität Rostock, 18055 Rostock and Fachbereich Mathematik, TU Berlin, 10623 Berlin, Germany (jungel@sun.math.uni-rostock.de).
} 
equation once assumed that the semiconductor device is in the low injection regime, i.e. for small absolute values of the applied voltage. In [24] it is shown that in the high injection regime diffusion terms are no longer linear. A useful choice for $\varphi$ is then $\varphi(s)=s^{\alpha}$ for $\alpha=\frac{5}{3}$ and $s \geq 0$. With this pressure function the parabolic equations in (1.1) become of degenerate type and existence of solutions does not follow from standard theory. In this paper we present results including both the low and high injection cases.

Function $\varphi$ may be interpreted in the language of gas dynamics. Assuming that particles behave, thermodynamically speaking, as an ideal gas satisfying the law $\varphi=$ $u \theta$, with $\theta$ the particle temperature, we obtain in the isothermal case a linear pressure term. However, in the isentropic case [10] temperature depends on concentrations as $\theta(u)=u^{2 / 3}$ and therefore $\varphi(u)=u^{5 / 3}$.

The standard or low injection model has been mathematically and numerically investigated in many papers, see [33], [34] and references therein. Existence and uniqueness of weak solutions was shown.

The isentropic or high injection model for linear $b$ and monotonic $F$, including the non-Lipschitz continuous case, has been analyzed in [22]-[25] where existence of weak solutions was proven. However, there is a lacking in results concerning the uniqueness of solutions when the problem actually degenerates. Besides, there are no results for general mobility functions.

As in the question of existence, the main difficulty to prove uniqueness of solutions relies in the simultaneous presence of a transport term and a nonlinear degenerate diffusion term. This kind of difficulty has already received the attention of many authors and has been solved for scalar equations of the type

$$
u_{t}-\operatorname{div}(\nabla \varphi(u)+b(u) \mathbf{e})=F(u)
$$

with e a prescribed vector field. The most successful technique developed to prove uniqueness of solutions of (1.2) is based on the use of the test function $\operatorname{sign}_{+}\left(u_{1}-u_{2}\right)$ in the weak formulation of (1.2), being $u_{1}$ and $u_{2}$ two, a priori, possible solutions of (1.2) in some sense. The core of the problem is to show that solutions have enough regularity to define the sign function as an admissible test function. This justification has been carried out by different means. One of them, introduced by Kruzhkov in [28] to prove an $L^{1}$ contraction property of entropy solutions of hyperbolic equations, is based in doubling the time variable and performing a passing to the limit in which these variables collapse. This technique has been applied to parabolic scalar equations, see, e.g., [29], [9], [18], [19], [35], and also to certain systems of parabolic equations coupled through reaction terms, but not through transport terms, see [36]. Notice that when applying succesfully this technique, uniqueness is always obtained as a by-product of a comparison principle. However, systems coupled through transport terms does not exhibit, in general, a comparison property. Therefore, other means have to be applied in order to give criteria to ensure uniqueness of solutions.

The outline of the paper is as follows. In Section 2 we present the assumptions on the data and the notion of weak solution. Then we prove Theorem 2.1 of existence of weak solutions by means of a regularization technique which involves the consideration of a non-degenerate problem for which existence of solutions is proven by a fixed point argument, see Theorem 2.2. In Section 3 we study the uniqueness of solutions and present three results, see Theorems 3.1, 3.3 and 3.4. The first result covers the situation in which diffusion dominates both transport and reaction. The second result only needs $\varphi$ non-decreasing, but an entropy type condition must hold for the electric 
field. Finally, the third result uses the assumption $\nabla u, \nabla v \in L^{1}\left(Q_{T}\right)$. We show that this regularity is achieved, at least, for data smooth enough and in space dimension one.

2. Existence of solutions. In this section we prove existence of weak solutions of problem (1.1). The main result is Theorem 2.1 where we prove existence in the most interesting case: when the parabolic equations of (1.1) are of degenerate type. As we already mentioned, the transport terms $\operatorname{div}(b(u) \nabla w)$ and $\operatorname{div}(b(v) \nabla w)$ are the main difficulty in the proof due to the fact that natural a priori estimates of problem (1.1) are obtained in terms of $\varphi(u)$ (with $\varphi^{\prime}(0)=0$ ) and their spatial derivatives meanwhile transport terms contain $b(u)$ and $b(v)$ which, in general, are not bounded by the former.

This difficulty leads us to consider an auxiliar non-degenerate problem for which we obtain existence of weak solutions, see Theorem 2.2 , and which allows us, by means of techniques of regularization and passing to the limit, to prove the result for the general formulation.

Before stating the first result we introduce a set of assumptions on the data as well as the definition of weak solution of (1.1).

Assumptions ON THE DATA.

$\mathbf{H}_{1} . \Omega \subset \mathbb{R}^{N}, N \leq 3$, is an open, bounded and connected set. The boundary of $\Omega$, $\partial \Omega$, is of class $\mathcal{C}^{1,1}$ and it splits in two disjoint components $\Gamma_{D}$, with positive measure, and $\Gamma_{N}$, open in $\partial \Omega$. We assume that for any function $\psi$ satisfying

$$
\left\{\begin{array}{l}
\Delta \psi \in L^{q}(\Omega) \\
\psi=0 \text { on } \Gamma_{D} \\
\nabla \psi \cdot \nu=0 \text { on } \Gamma_{N}
\end{array}\right.
$$

we have the regularity $\psi \in W^{2, q}(\Omega)$, for $q \in[1, \infty)$. Finally, we suppose $T>0$ is arbitrarily chosen.

Definition. A function $f: \mathbb{R}^{n} \rightarrow \mathbb{R}$ is sublinear if there exists a positive constant c such that

$$
\left|f\left(s_{1}, \ldots, s_{n}\right)\right| \leq c\left(1+\sum_{i=1}^{n}\left|s_{i}\right|\right), \quad \forall\left(s_{1}, \ldots, s_{n}\right) \in \mathbb{R}^{n}
$$

$\mathbf{H}_{2}$. We assume

$$
\begin{gathered}
\varphi \in \mathcal{C}([0, \infty)) \cap \mathcal{C}^{1}((0, \infty)), \quad \varphi^{\prime}(0)=0, \quad \varphi \text { non-decreasing, } \\
F \in \mathcal{C}_{l o c}^{0,1}\left([0, \infty)^{2} ; \mathbb{R}\right), \\
b \in \mathcal{C}^{1}([0, \infty)) \text { is sublinear and satisfies } \\
\left|b^{\prime}(s)\right| \leq c\left(1+\varphi^{\prime}(s)\right), \quad \text { for all } s \in[0, \infty)
\end{gathered}
$$

and for some constant $c>0$.

$\mathbf{H}_{3}$. The auxiliary data satisfy

$$
\begin{aligned}
& u_{0}, v_{0} \in L^{\infty}(\Omega), \quad u_{0} \geq 0, \quad v_{0} \geq 0 \quad \text { in } \Omega \\
& \varphi\left(u_{D}\right), \varphi\left(v_{D}\right) \in L^{\infty}\left(0, T ; H^{1}(\Omega)\right) \cap L^{\infty}\left(Q_{T}\right) \cap H^{1}\left(0, T ; L^{2}(\Omega)\right), \\
& w_{D} \in L^{\infty}\left(0, T ; W^{2, \infty}(\Omega)\right) .
\end{aligned}
$$


Although physically $C=C(x)$, we shall consider the more general case $C=C(x, t)$ under the assumption $C \in L^{\infty}\left(Q_{T}\right)$.

$\mathbf{H}_{4}$. If both $F$ and $b$ are nonlinear then we assume $\varphi^{-1} \in \mathcal{C}^{0, \alpha}([0, \infty))$, for some $\alpha \in(0,1)$.

We remark that the property assumed for problem (2.1) actually represents a condition on the contact angles of the boundary segments $\Gamma_{D}$ and $\Gamma_{N}$, see, e.g. [32]. In particular, if both components of the boundary are open and closed, so they do not meet, then the assumption is a well known result, see, e.g. [41]. As stated in (2.2), in this article we shall consider a Lipschitz continuous recombination-generation term $F$. The case of a monotone $F$ was already treated in [24] obtaining similar results on the existence of weak solutions under somehow stronger conditions on $\varphi$ and $b$. As shown in [12], a monotone non-Lipschitz continuous recombination-generation term may imply the formation of dead cores (sets where the components $u, v$ of the solution vanish even when the initial data are strictly positive) and play an important role in applications through the phenomenon known as vacuum solutions, see [12].

We consider a notion of weak solution similar to that introduced in [1]:

Definition of Weak solution. Set

$$
\mathcal{V}:=\left\{z \in H^{1}(\Omega): z=0 \quad \text { on } \quad \Gamma_{D}\right\},
$$

and assume $\mathbf{H}_{1}-\mathbf{H}_{3}$. Then $(u, v, w)$ is a weak solution of (1.1) if the following properties hold:

(i) $u, v \in L^{\infty}\left(Q_{T}\right), \varphi(u) \in \varphi\left(u_{D}\right)+L^{2}(0, T ; \mathcal{V}), \varphi(v) \in \varphi\left(v_{D}\right)+L^{2}(0, T ; \mathcal{V})$ and $w \in w_{D}+L^{2}(0, T ; \mathcal{V}) \cap L^{\infty}\left(Q_{T}\right)$.

(ii)

$$
\begin{aligned}
& \int_{0}^{T}\left\langle u_{t}, \zeta\right\rangle+\int_{0}^{T} \int_{\Omega}(\nabla \varphi(u)-b(u) \nabla w) \cdot \nabla \zeta=\int_{0}^{T} \int_{\Omega} F(u, v) \zeta, \\
& \int_{0}^{T}\left\langle v_{t}, \zeta\right\rangle+\int_{0}^{T} \int_{\Omega}(\nabla \varphi(v)-b(v) \nabla w) \cdot \nabla \zeta=\int_{0}^{T} \int_{\Omega} F(u, v) \zeta, \\
& \int_{0}^{T} \int_{\Omega} \nabla w \cdot \nabla \zeta=\int_{0}^{T} \int_{\Omega}(v-u-C) \zeta
\end{aligned}
$$

for any test function $\zeta \in L^{2}(0, T ; \mathcal{V})$ (notice that due to $(2.2) F(u, v) \in L^{2}\left(Q_{T}\right)$ ).

(iii) $u_{t}, v_{t} \in L^{2}\left(0, T ; \mathcal{V}^{\prime}\right)$ and the initial data are verified in the following sense:

$$
\begin{aligned}
& \int_{0}^{T}\left\langle u_{t}, \zeta\right\rangle+\int_{0}^{T} \int_{\Omega}\left(u-u_{0}\right) \zeta_{t}=0, \\
& \int_{0}^{T}\left\langle v_{t}, \zeta\right\rangle+\int_{0}^{T} \int_{\Omega}\left(v-v_{0}\right) \zeta_{t}=0,
\end{aligned}
$$

for any test function $\zeta \in L^{2}(0, T ; \mathcal{V}) \cap W^{1,1}\left(0, T ; L^{2}(\Omega)\right)$ with $\zeta(T)=0$.

Next we state the main result of this section:

TheOREM 2.1. Assume $\mathbf{H}_{1}-\mathbf{H}_{4}$ and suppose that the auxiliary data satisfy

$$
\begin{gathered}
k \geq u_{0}, v_{0} \geq m \geq 0 \quad \text { in } \Omega \quad \text { and } \\
\varphi\left(k e^{\lambda_{0} t}\right) \geq \varphi\left(u_{D}\right), \varphi\left(v_{D}\right) \geq \varphi\left(m e^{-\lambda_{1} t}\right) \geq 0 \quad \text { in } \Sigma_{D},
\end{gathered}
$$

for some non-negative constants $k, m, \lambda_{0}, \lambda_{1}$. Then there exists $\lambda \geq 0$, independent of $\varphi$, such that problem (1.1) has a weak solution verifying

$$
k e^{\lambda t} \geq u(\cdot, t), v(\cdot, t) \geq m e^{-\lambda t} \geq 0 \quad \text { in } \Omega \text { for a.e. } t \in(0, T),
$$




$$
\begin{aligned}
& u, v \in \mathcal{C}\left([0, T] ; \mathcal{V}^{\prime}\right), \\
& w \in L^{\infty}\left(0, T ; W^{2, s}(\Omega)\right) \quad \text { for all } s \in[1, \infty) .
\end{aligned}
$$

Moreover, if $\varphi \in \mathcal{C}^{1}([0, \infty))$ then

$$
\sqrt{\varphi^{\prime}(u)} \nabla u, \sqrt{\varphi^{\prime}(v)} \nabla v \in L^{2}\left(Q_{T}\right) .
$$

The proof is based on the following previous result for the non-degenerate problem:

Theorem 2.2. Assume $\mathbf{H}_{1}-\mathbf{H}_{3}$ and let $\varphi$ be sublinear and strictly increasing. Suppose $\varphi^{-1}$ Lipschitz continuous, $F$ sublinear and auxiliary data satisfaying (2.6) for some non-negative constants $k, m, \lambda_{0}, \lambda_{1}$. Then there exists $\lambda \geq 0$, independent of $\varphi$, such that problem (1.1) has a weak solution verifying (2.7), (2.8) and

$$
u, v \in \mathcal{C}\left([0, T] ; L^{1}(\Omega)\right) .
$$

Proof of Theorem 2.2 is based on a fixed point technique. To define the fixed point operator in $L^{p}$ spaces we need, due to the lack of regularity of the term $\nabla b(u) \cdot \nabla w$, to uncouple problem (1.1) and to consider two auxiliary problems, see (2.13) and (2.14). First we apply a fixed point argument to obtain the existence of solutions, $(u, v)$, of (2.13) and we show that this solution satisfies (2.7). Then, we solve problem (2.14) and use again a fixed point argument to couple the whole system, obtaining in this way a weak solution of (1.1) with the property (2.7). The additional regularity is obtained by applying general results on $L^{p}$ spaces, see [39].

Proof of Theorem 2.2

We start introducing some notation and an elementary consequence of Sobolev's theorem: we shall write $\|\cdot\|_{L^{p}}:=\|\cdot\|_{L^{p}\left(Q_{T}\right)},\|\cdot\|_{L^{p}\left(L^{q}\right)}:=\|\cdot\|_{L^{p}\left(0, T ; L^{q}(\Omega)\right)}$ and

$$
\||f|\|:=\|f\|_{L^{\infty}\left(L^{2}\right)}+\|f\|_{L^{2}(0, T ; \mathcal{V})} .
$$

Let $2^{*}$ be the critical Sobolev exponent given by $\infty, s \in[1, \infty)$ or 6 if $N=1,2,3$ respectively. Then we have that for all $f \in L^{\infty}\left(0, T ; L^{2}(\Omega)\right) \cap L^{2}(0, T ; \mathcal{V})$ there exists a positive constant $c \equiv c(\Omega)$ such that

$$
\|f\|_{L^{r}} \leq c\||f|\|, \quad \text { with } \quad 1 \leq r<4\left(1-\frac{1}{2^{*}}\right) .
$$

Finally, $c$ shall denote a positive constant independent of the relevant quantities of

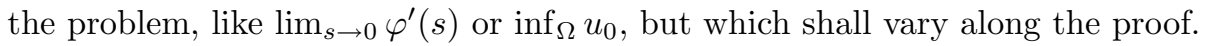

Step 1. Let $T>0,0<\rho<c_{\rho}$, with $c_{\rho}$ a positive constant to be fixed, and fix $r$ such that

$$
3<r<4\left(1-\frac{1}{2^{*}}\right)
$$

Let $p$ be an exponent satisfying the following restriction:

$$
\frac{r}{r-2}<3<p<r
$$

with $r$ given by (2.10). Consider the set $K \subset L^{p}\left(0, T ; W^{2, p}(\Omega)\right) \cap L^{2}(0, T ; \mathcal{V})$ given by

$$
h \in K \Leftrightarrow \Delta h \in L^{\infty}\left(Q_{T}\right) \text { and }\|\Delta h\|_{L^{p}}+\|\nabla h\|_{L^{2}} \leq \rho .
$$


Clearly, $K$ is convex. Moreover, since due to the choice of $p$ we have $2 \leq \frac{N p}{N-p}$, it follows that $\|\Delta w\|_{L^{p}}+\|\nabla w\|_{L^{2}}$ is a norm in $L^{p}\left(0, T ; W^{2, p}(\Omega)\right) \cap L^{2}(0, T ; \mathcal{V})$ and therefore $K$ is weakly compact in this space. These properties of $K$ will be used later to apply a fixed point argument. Given $h \in K$ we introduce problems

$$
\begin{cases}u_{t}-\operatorname{div}(\nabla \varphi(u)-b(u) \nabla h)=F(u, v) & \text { in } Q_{T}, \\ v_{t}-\operatorname{div}(\nabla \varphi(v)+b(v) \nabla h)=F(u, v) & \text { in } Q_{T}, \\ \nabla \varphi(u) \cdot \nu=0, \quad \nabla \varphi(v) \cdot \nu=0, & \text { on } \Sigma_{N}, \\ \varphi(u)=\varphi\left(u_{D}\right), \quad \varphi(v)=\varphi\left(v_{D}\right), & \text { on } \Sigma_{D} \\ u(\cdot, 0)=u_{0}, \quad v(\cdot, 0)=v_{0}, & \text { in } \Omega\end{cases}
$$

and

$$
\begin{cases}-\Delta w=v-u-C & \text { in } Q_{T}, \\ w=w_{D} & \text { on } \Sigma_{D} \\ \nabla w \cdot \nu=0 & \text { on } \Sigma_{N}\end{cases}
$$

with similar notions of weak solutions as for problem (1.1).

Step 2. Definition of the fixed point operator for (2.13). Consider problems

$$
\begin{cases}u_{t}-\Delta \varphi(u)=f & \text { in } Q_{T}, \\ \varphi(u)=\varphi\left(u_{D}\right) & \text { on } \Gamma_{D}, \\ \nabla \varphi(u) \cdot \nu=0 & \text { on } \Gamma_{N}, \\ u(\cdot, 0)=u_{0} & \text { in } \Omega,\end{cases}
$$

and

$$
\left\{\begin{array}{lc}
v_{t}-\Delta \varphi(v)=g & \text { in } Q_{T}, \\
\varphi(v)=\varphi\left(v_{D}\right) & \text { on } \Gamma_{D}, \\
\nabla \varphi(v) \cdot \nu=0 & \text { on } \Gamma_{N}, \\
v(\cdot, 0)=v_{0} & \text { in } \Omega,
\end{array}\right.
$$

with $f, g \in L^{2}\left(Q_{T}\right)$. Since these problems are uniformly parabolic we deduce, see e.g. [30], the existence of a unique weak solution of (2.15) and (2.16) with the regularity $u, v \in L^{r}\left(Q_{T}\right) \cap \mathcal{C}\left([0, T] ; L^{1}(\Omega)\right), \varphi(u) \in \varphi\left(u_{D}\right)+L^{2}(0, T ; \mathcal{V}), \varphi(v) \in$ $\varphi\left(v_{D}\right)+L^{2}(0, T ; \mathcal{V})$, with $r$ given by $(2.10)$.

We introduce the set

$$
K^{*}:=\left\{(f, g) \in L^{2}\left(Q_{T^{*}}\right) \times L^{2}\left(Q_{T^{*}}\right):\|f\|_{L^{2}},\|g\|_{L^{2}}<R\right\}, \quad 0<T^{*} \leq T,
$$

which is convex and weakly compact in $L^{2}\left(Q_{T^{*}}\right) \times L^{2}\left(Q_{T^{*}}\right)$, and the mapping $Q$ : $K^{*} \rightarrow L^{2}\left(Q_{T^{*}}\right) \times L^{2}\left(Q_{T^{*}}\right)$ given by

$$
Q(f, g):=(F(u, v)-\operatorname{div}(b(u) \nabla h), F(u, v)+\operatorname{div}(b(v) \nabla h)),
$$

with $u, v$ solutions of (2.15), (2.16). It can be shown that, as a consequence of (2.3) and the sublinearity of $F$ and $b$, the operator $Q$ is well defined. Notice also that a fixed point of $Q$ is a weak local solution of (2.13). To prove the existence of such a point we search for $R$ and $T^{*}$ such that

(i) $Q\left(K^{*}\right) \subset K^{*}$, and

(ii) $Q$ is weakly-weakly sequentially continuous in $L^{2}\left(Q_{T^{*}}\right) \times L^{2}\left(Q_{T^{*}}\right)$, 
which will allow us to apply the fixed point theorem of [4] to deduce the result. Since problems (2.15) and (2.16) share the same structure we shall only work out the properties satisfied by solutions of (2.15).

Step 3. A priori estimates for problems (2.15) and (2.16). Proof of $Q\left(K^{*}\right) \subset K^{*}$. This last condition reads as

$$
I_{u}:=\|F(u, v)-\nabla b(u) \cdot \nabla h-b(u) \Delta h\|_{L^{2}} \leq R .
$$

Taking $\zeta=\varphi(u)-\varphi\left(u_{D}\right)$ as a test function for problem (2.15) we get

$$
\begin{aligned}
\int_{\Omega} \Phi(u(t))+\int_{Q_{T}}|\nabla \varphi(u)|^{2}= & \int_{\Omega} \Phi\left(u_{0}\right)-\int_{Q_{T}}\left(u-u_{0}\right) \varphi\left(u_{D}\right)_{t}+\int_{\Omega}\left(u(t)-u_{0}\right) \varphi\left(u_{D}\right) \\
& +\int_{Q_{T}} \nabla \varphi(u) \cdot \nabla \varphi\left(u_{D}\right)+\int_{Q_{T}} f\left(\varphi(u)-\varphi\left(u_{D}\right)\right),
\end{aligned}
$$

with $\Phi(s):=\int_{0}^{s} \varphi(\sigma) d \sigma$. Using $\varphi^{-1}$ Lipschitz continuous and standard inequalities we get from $(2.18)$

$$
c_{\varphi}\left(\|u\|_{L^{\infty}\left(L^{2}\right)}^{2}+\|\nabla u\|_{L^{2}}^{2}\right) \leq\|\Phi(u)\|_{L^{\infty}\left(L^{1}\right)}+\|\nabla \varphi(u)\|_{L^{2}}^{2} \leq \Lambda+\|f\|_{L^{2}}^{2},
$$

with $c_{\varphi}, \Lambda$ constants depending on the Lipschitz continuity constant of $\varphi^{-1}$ and on the auxiliary data, respectively. Using (2.9) and $f \in K^{*}$ we deduce

$$
\|u\|_{L^{r}} \leq c\|\| u\|\| c(\Lambda+R) .
$$

Since $r>2$ we have

$$
\|u\|_{L^{2}} \leq A_{0}\left(T^{*}\right)\|u\|_{L^{r}} \leq c A_{0}\left(T^{*}\right)(\Lambda+R),
$$

with $A_{0}\left(T^{*}\right):=\left|Q_{T^{*}}\right|^{\frac{r-2}{2 r}}$. Finally, since $\varphi$ is sublinear we deduce the existence of a continuous non-decreasing function $\eta:(0, \infty) \rightarrow(0, \infty)$ such that

$$
\|\nabla \varphi(u)\|_{L^{2}}^{2} \leq \Lambda+\eta\left(T^{*}\right)
$$

with $\eta\left(T^{*}\right) \rightarrow 0$ as $T^{*} \rightarrow 0$, see [16]. We are now ready to estimate the terms in (2.17): $F$ sublinear and (2.21) imply

$$
\|F(u, v)\|_{L^{2}} \leq c A_{1}\left(T^{*}\right),
$$

with $A_{1}\left(T^{*}\right):=A_{0}\left(T^{*}\right)(\Lambda+2 R)+\left|Q_{T^{*}}\right|^{1 / 2}$. From (2.3), (2.22) and the regularity of $h$ we get

$$
\|\nabla b(u) \cdot \nabla h\|_{L^{2}} \leq c\|\nabla \varphi(u)\|_{L^{2}}\|\nabla h\|_{L^{\infty}} \leq c\left(\Lambda+\eta\left(T^{*}\right)\right)\|\nabla h\|_{L^{\infty}},
$$

and since $b$ is sublinear

$$
\|b(u) \Delta h\|_{L^{2}} \leq\|b(u)\|_{L^{2}}\|\Delta h\|_{L^{\infty}} \leq c A_{2}\left(T^{*}\right)\|\Delta h\|_{L^{\infty}},
$$

with $A_{2}\left(T^{*}\right):=\left|Q_{T^{*}}\right|^{\frac{1}{2}}+\left|Q_{T^{*}}\right|^{\frac{r-2}{2 r}}(\Lambda+R)$. Gathering (2.23), (2.24) and (2.25) we obtain

$$
I_{u} \leq c A_{1}\left(T^{*}\right)+c\left(\Lambda+\eta\left(T^{*}\right)\right)\|\nabla h\|_{L^{\infty}}+c A_{2}\left(T^{*}\right)\|\Delta h\|_{L^{\infty}},
$$


and since we want $I_{u} \leq R$, it is sufficient to make

$$
c A_{1}\left(T^{*}\right)+c\left(\Lambda+\eta\left(T^{*}\right)\right)\|\nabla h\|_{L^{\infty}}+c A_{2}\left(T^{*}\right)\|\Delta h\|_{L^{\infty}} \leq R .
$$

Since $A_{1}, A_{2}, \eta$ are non-decreasing continuous functions in $\mathbb{R}_{+}$, we have that, fixing $R$ such that

$$
R>c A_{1}(T)+c\left(\Lambda\left(u_{0}, \varphi\left(u_{D}\right)\right)+\eta(T)\right)\|\nabla h\|_{L^{\infty}\left(Q_{T}\right)}+c A_{2}(T)\|\Delta h\|_{L^{\infty}\left(Q_{T}\right)}
$$

inequality (2.26) is satisfied for all $T^{*} \in[0, T]$. An identical argument allows us to get $I_{v} \leq R$. Therefore, we have proven the existence of $R$ and $T^{*}$, which can be taken as $T^{*}=T$, such that $Q\left(K^{*}\right) \subset K^{*}$.

Step 4. Continuity of $Q$. Consider any sequence $\left(f_{j}, g_{j}\right) \subset K^{*} \rightarrow(f, g)$ weakly in $L^{2}\left(Q_{T}\right) \times L^{2}\left(Q_{T}\right)$ and let us show

$$
\begin{array}{ll}
\operatorname{div}\left(b\left(u_{j}\right) \nabla h\right) \rightarrow \operatorname{div}(b(u) \nabla h) & \text { weakly in } L^{2}\left(Q_{T}\right), \\
F\left(u_{j}, v_{j}\right) \rightarrow F(u, v) & \text { weakly in } L^{2}\left(Q_{T}\right),
\end{array}
$$

with $u_{j}, v_{j}, u, v$ solutions of $(2.15)$, (2.16) corresponding to $f_{j}, g_{j}, f, g$, respectively. By $(2.20)$ we have $\left\|u_{j}\right\|_{L^{r}} \leq c\left\|\left|u_{j}\right|\right\| \leq$ const., and from (2.15) we also deduce $\left\|u_{j t}\right\|_{L^{2}\left(0, T ; \mathcal{V}^{\prime}\right)} \leq$ const. Passing to a subsequence, if necessary, we obtain

$$
\begin{array}{ll}
u_{j} \rightarrow u & \text { weakly in } L^{r}\left(Q_{T}\right), \\
u_{j} \rightarrow u & \text { strongly in } L^{2}\left(Q_{T}\right) \text { and a.e. in } Q_{T}, \\
\nabla u_{j} \rightarrow \nabla u & \text { weakly in } L^{2}\left(Q_{T}\right) \\
u_{j t} \rightarrow u_{t} & \text { weakly in } L^{2}\left(0, T ; \mathcal{V}^{\prime}\right) .
\end{array}
$$

Since $F$ is sublinear and $u_{j}, v_{j}$ are bounded in $L^{r}\left(Q_{T}\right)$ it follows $\left\|F\left(u_{j}, v_{j}\right)\right\|_{L^{r}} \leq$ const. and then $F\left(u_{j}, v_{j}\right) \rightarrow \tilde{F}$ in $L^{r}\left(Q_{T}\right)$ for some $\tilde{F} \in L^{r}\left(Q_{T}\right)$. Continuity of $F$ together with pointwise convergence of $u_{j}, v_{j}$ imply that $F\left(u_{j}, v_{j}\right) \rightarrow F(u, v)$ a.e. in $Q_{T^{*}}$, and therefore $\tilde{F} \equiv F(u, v)$. Hence, $F\left(u_{j}, v_{j}\right) \rightarrow F(u, v)$ strongly in $L^{r}\left(Q_{T}\right)$. A similar argument shows that $b\left(u_{j}\right) \rightarrow b(u)$ strongly in $L^{r}\left(Q_{T}\right)$. Finally, since $r>2$ and $\nabla h \in L^{\infty}\left(Q_{T}\right)$ we deduce $\operatorname{div}\left(b\left(u_{j}\right) \nabla h\right) \rightarrow \operatorname{div}(b(u) \nabla h)$ weakly in $L^{2}\left(Q_{T}\right)$. Hence, $Q$ is weakly-weakly sequentially continuous. By the fixed point theorem [4] we deduce the existence of a weak solution $(u, v)$ of $(2.13)$ with the same regularity obtained for the solutions of (2.15) and (2.16) when $f, g \in L^{2}\left(Q_{T}\right)$ is assumed. Notice that the solution found is global in time.

Step 5. Lower bound and $L^{\infty}$ regularity of $u, v$. We introduce in problem (2.13) the change of unknowns $U:=u e^{-\beta t}$ and $V:=v e^{-\beta t}$ with $\beta>0$. Then $(U, V)$ satisfies

$$
\begin{cases}U_{t}+\beta U-e^{-\beta t} d i v\left(\nabla \varphi\left(e^{\beta t} U\right)-b\left(e^{\beta t} U\right) \nabla h\right)=\hat{F} & \text { in } Q_{T}, \\ V_{t}+\beta V-e^{-\beta t} d i v\left(\nabla \varphi\left(e^{\beta t} V\right)+b\left(e^{\beta t} V\right) \nabla h\right)=\hat{F} & \text { in } Q_{T}, \\ \nabla \varphi\left(e^{\beta t} U\right) \cdot \nu=0, \quad \nabla \varphi\left(e^{\beta t} V\right) \cdot \nu=0, & \text { on } \Sigma_{N} \\ \varphi\left(e^{\beta t} U\right)=\varphi\left(u_{D}\right), \quad \varphi\left(e^{\beta t} V\right)=\varphi\left(v_{D}\right), & \text { on } \Sigma_{D} \\ U(\cdot, 0)=u_{0}, \quad V(\cdot, 0)=v_{0}, & \text { in } \Omega,\end{cases}
$$

with $\hat{F}:=e^{-\beta t} F\left(e^{\beta t} U, e^{\beta t} V\right)$. To obtain the lower bound we compare $U$ and $V$ with $z(t):=m e^{-(\lambda+\beta) t}$, for a suitable $\lambda>\lambda_{1}$. By assumption, $u_{D} \geq m e^{-\lambda_{1} t} \geq m e^{-\lambda t}$ and 
then we can take $Z_{u}:=\min \{U-z, 0\}$ as test function obtaining

$$
\begin{aligned}
\int_{\Omega} Z_{u}(U-z)_{t}- & \lambda \int_{\Omega} z Z_{u}+\beta \int_{\Omega} Z_{u}^{2}+e^{-\beta t} \int_{\Omega} \nabla \varphi\left(e^{\beta t} U\right) \cdot \nabla Z_{u} \\
= & -e^{-\beta t} \int_{\Omega} Z_{u}\left[\nabla b\left(e^{\beta t} U\right) \cdot \nabla h+b\left(e^{\beta t} U\right) \Delta h\right] \\
& +e^{-\beta t} \int_{\Omega} Z_{u} F\left(e^{\beta t} U, e^{\beta t} V\right) .
\end{aligned}
$$

Since $b$ is Lipschitz continuous (with constant $M_{b}$ ) by estimating

$$
\int_{\Omega} Z_{u} b^{\prime}\left(e^{\beta t} U\right) \nabla Z_{u} \cdot \nabla h \leq M_{b} \int_{\Omega}\left|\nabla Z_{u}\right|^{2}+M_{b}\|\nabla h\|_{L^{\infty}}^{2} \int_{\Omega} Z_{u}^{2}
$$

and

$$
\begin{aligned}
\int_{\Omega} Z_{u} b\left(e^{\beta t} U\right) \Delta h & =\int_{\Omega} Z_{u}\left(b\left(e^{\beta t} U\right)-b\left(e^{\beta t} z\right)+b\left(e^{\beta t} z\right)\right) \Delta h \\
& \leq c e^{\beta t} M_{b}\|\Delta h\|_{L^{\infty}}\left(\int_{\Omega} Z_{u}^{2}+\int_{\Omega} z\left|Z_{u}\right|\right)
\end{aligned}
$$

we obtain from $(2.27)$

$$
\frac{d}{d t} \int_{\Omega} Z_{u}^{2}+\lambda^{\prime} \int_{\Omega} z\left|Z_{u}\right|+\beta^{\prime} \int_{\Omega} Z_{u}^{2} \leq e^{-\beta t} \int_{\Omega} Z_{u} F\left(e^{\beta t} U, e^{\beta t} V\right),
$$

with $\lambda^{\prime}:=\lambda-c M_{b}\|\Delta h\|_{L^{\infty}}, \beta^{\prime}:=\beta-M_{b}\|\nabla h\|_{L^{\infty}}^{2}-c M_{b}\|\Delta h\|_{L^{\infty}}$, where we used $-\lambda Z_{u}=\lambda\left|Z_{u}\right|$. Since $F$ is Lipschitz continuous we can use a similar argument to show that

$$
Z_{u} F\left(e^{\beta t} U, e^{\beta t} V\right) \leq c Z_{u}\left(Z_{u}+Z_{v}+F\left(e^{\beta t} z, e^{\beta t} z\right)\right),
$$

with $Z_{v}:=\min \{V-z, 0\}$. Adding to (2.28) the similar estimate for $V$ we get in the right hand side of the resulting inequality the term

$$
e^{-\beta t} \int_{\Omega} F\left(e^{\beta t} U, e^{\beta t} V\right)\left(Z_{u}+Z_{v}\right)
$$

Using (2.29) and the similar estimate for $Z_{v} F\left(e^{\beta t} U, e^{\beta t} V\right)$ (2.30) may be estimated as

$$
e^{-\beta t} \int_{\Omega} F\left(e^{\beta t} U, e^{\beta t} V\right)\left(Z_{u}+Z_{v}\right) \leq c \int_{\Omega}\left(Z_{u}^{2}+Z_{v}^{2}+z\left|Z_{u}\right|+z\left|Z_{v}\right|\right) .
$$

Then, for $\beta \geq M_{b}\|\nabla h\|_{L^{\infty}}^{2}+c M_{b}\|\Delta h\|_{L^{\infty}}+c$ and $\lambda \geq c M_{b}\|\Delta h\|_{L^{\infty}}+c$ we obtain

$$
\frac{d}{d t} \int_{\Omega}\left(Z_{u}^{2}+Z_{v}^{2}\right) \leq 0
$$

from where the result follows. Notice that neither $\beta$ nor $\lambda$ depend on $\varphi$. Finally, the estimate $u, v \in L^{\infty}\left(Q_{T}\right)$ is obtained using a similar technique and we omit therefore the proof, see [20] for details.

Step 6. End of proof of existence of local solutions of (1.1). Let $\tilde{T} \in(0, T]$ to be fixed, $\tilde{K}$ defined by $(2.12)$ with $T$ replaced by $\tilde{T}, h \in \tilde{K}$ and $u, v$ be solutions of 
(2.15), (2.16) corresponding to $h$. Consider problem (2.14) in $Q_{\tilde{T}}$. Since $u, v, C \in$ $L^{\infty}\left(Q_{\tilde{T}}\right)(2.14)$ has a unique solution $w \in L^{\infty}\left(0, \tilde{T}, W^{2, s}(\Omega)\right) \cap L^{2}(0, T ; \mathcal{V})$ with $\Delta w \in$ $L^{\infty}\left(Q_{\tilde{T}}\right)$. In particular, $w \in L^{p}\left(0, \tilde{T}, W^{2, p}(\Omega)\right) \cap L^{2}(0, T ; \mathcal{V})$. We define $P: \tilde{K} \rightarrow$ $L^{p}\left(0, \tilde{T}, W^{2, p}(\Omega)\right) \cap L^{2}(0, T ; \mathcal{V})$ by $P(h)=w$, being $w$ such solution. Notice that if $w$ is a fixed point for $P$ then $(u, v, w)$ is a local solution of (1.1). To prove the existence of a fixed point we use the same technique than before, which consists on showing

(i) $P(\tilde{K}) \subset \tilde{K}$, i.e., $\Delta w \in L^{\infty}\left(Q_{T}\right)$, and $\|\Delta w\|_{L^{p}}+\|\nabla w\|_{L^{2}}<\rho$ and

(ii) $P$ is weakly-weakly sequentially continuous in $L^{p}\left(0, \tilde{T}, W^{2, p}(\Omega)\right) \cap L^{2}(0, T ; \mathcal{V})$.

From (2.14):

$$
\|\Delta w\|_{L^{s}} \leq\|u\|_{L^{s}}+\|v\|_{L^{s}}+\|C\|_{L^{s}}, \quad \text { for all } s \in[1, \infty] .
$$

Multiplying the equation in (2.14) by $w-w_{D}$ and using Hölder and Poincaré's inequalities we obtain

$$
\|\nabla w\|_{L^{2}} \leq c\left(\|u\|_{L^{2}}+\|v\|_{L^{2}}+\|C\|_{L^{2}}+\left\|w_{D}\right\|_{L^{2}}+\left\|\nabla w_{D}\right\|_{L^{2}}\right) .
$$

From (2.31), (2.32) and $p>2$ we get

$$
(2.33)\|\Delta w\|_{L^{p}}+\|\nabla w\|_{L^{2}} \leq c\left(\|u\|_{L^{p}}+\|v\|_{L^{p}}+\|C\|_{L^{p}}+\left\|w_{D}\right\|_{L^{2}}+\left\|\nabla w_{D}\right\|_{L^{2}}\right) .
$$

By (2.11) we have $p<r$ and therefore

$$
\|u\|_{L^{p}} \leq A(\tilde{T})\|u\|_{L^{r}} \leq c A(\tilde{T})\||u|\|,
$$

with $A(\tilde{T}):=\left|Q_{\tilde{T}}\right|^{\frac{r p}{r-p}}$. Assume that the estimate

$$
\||u|\| \leq G(\rho, \tilde{T})
$$

holds, with $G$ continuous, bounded as a function of $\tilde{T}$ and increasing with respect to $\rho$ in an interval $\left(0, c_{\rho}\right)$, with $c_{\rho}>0$. We shall prove this estimate later on, see Step 7. Then, from (2.34) $\|u\|_{L^{p}} \leq c A(\tilde{T}) G(\rho, \tilde{T})$. A similar estimate holds for $v$. Since $C \in L^{\infty}\left(Q_{\tilde{T}}\right)$ and $w_{D} \in L^{\infty}\left(0, T ; H^{1}(\Omega)\right)$ we have $\|C\|_{L^{p}}+\left\|w_{D}\right\|_{L^{2}}+\left\|\nabla w_{D}\right\|_{L^{2}}=$ $B_{0}(\tilde{T})$ for a non-decreasing continuous function $B_{0}$ satisfying $B_{0}(0)=0$. From (2.33) we deduce

$$
\|\Delta w\|_{L^{p}}+\|\nabla w\|_{L^{2}} \leq A(\tilde{T}) G(\rho, \tilde{T})+B_{0}(\tilde{T}),
$$

and since we want to make $\|\Delta w\|_{L^{p}}+\|\nabla w\|_{L^{2}} \leq \rho$, it suffices to find $\tilde{T}>0$ such that

$$
A(\tilde{T}) G(\rho, \tilde{T})+B_{0}(\tilde{T})=\rho .
$$

Since $G$ is bounded as a function of $\rho$ and $A(\tilde{T}), B_{0}(\tilde{T}) \downarrow 0$ as $\tilde{T} \downarrow 0$ it is straightforward to see that such $\tilde{T}$ exists, so (i) is satisfied.

To prove the continuity we consider a sequence $h_{n} \in \tilde{K}$ such that $h_{n} \rightarrow h$ weakly in $L^{p}\left(0, \tilde{T} ; W^{2, p}(\Omega)\right) \cap L^{2}(0, T ; \mathcal{V})$ and we show that $w_{n} \rightarrow w$ weakly in $L^{p}\left(0, \tilde{T} ; W^{2, p}(\Omega)\right) \cap L^{2}(0, T ; \mathcal{V})$, with $w_{n}, w$ solutions of (2.14) corresponding to $h_{n}, h$. Since $h_{n} \in \tilde{K},\left\|\Delta h_{n}\right\|_{L^{p}}+\|\nabla w\|_{L^{2}} \leq \rho<c_{\rho}$, and then from (2.35) we get $\left\|\left|u_{n}\right|\right\|,\left\|\left|v_{n}\right|\right\|$ $\leq G\left(c_{\rho}, \tilde{T}\right) \leq$ const. Using $(2.9)$ we also obtain $\left\|u_{n}\right\|_{L^{r}},\left\|v_{n}\right\|_{L^{r}} \leq$ const. Then $u_{n} \rightarrow u$ weakly in $L^{r}\left(Q_{\tilde{T}}\right)$, and similarly for $v_{n}$. Since $p<r$ we find $\Delta w_{n} \rightarrow \Delta w$ weakly in $L^{p}\left(Q_{\tilde{T}}\right)$. From $r>2$ we also have $w_{n} \rightarrow w$ weakly in $L^{2}(0, T ; \mathcal{V})$. We deduce from 
the fixed point theorem [4] that $P$ has a fixed point, $(u, v, w)$, which is a weak local solution of (1.1) in $Q_{\tilde{T}}$ with the regularity inherited from problems (2.13) and (2.14). Moreover, since the estimates do not blow up when $\tilde{T}$ increases, see Step 7, we may use a standard continuation argument to deduce that the solution is global in time.

Step 7. Estimating $\||u|\|^{2}+\||v|\|^{2}$ of problem (2.13). Taking $\varphi(u)-\varphi\left(u_{D}\right)$ as test function for (2.13) and reasoning as in (2.18) with $f:=F(u, v)-\operatorname{div}(b(u) \nabla h)$ we get

$$
\begin{aligned}
\|\Phi(u)\|_{L^{\infty}\left(L^{1}\right)}+\|\nabla \varphi(u)\|_{L^{2}}^{2} \leq & \Lambda+\int_{Q_{\tilde{T}}} F(u, v)\left(\varphi(u)-\varphi\left(u_{D}\right)\right) \\
& +\int_{Q_{\tilde{T}}} b(u) \nabla h \cdot \nabla\left(\varphi(u)-\varphi\left(u_{D}\right)\right),
\end{aligned}
$$

with $\Lambda$ depending only on the auxiliary data. Since $F$ is sublinear we again obtain (2.23). Defining $B(s):=b(s) \varphi(s)-\int_{0}^{s} b^{\prime}(\sigma) \varphi(\sigma) d \sigma$ and using the sublinearity of $\varphi$ and $b$ and (2.3) we get $|B(s)| \leq c\left(1+|s|+s^{2}\right)$. Then

$$
\begin{aligned}
\int_{Q_{\tilde{T}}} b(u) \nabla h \cdot\left(\nabla \varphi(u)-\nabla \varphi\left(u_{D}\right)\right)= & -\int_{Q_{\tilde{T}}}\left(B(u)-B\left(u_{D}\right)\right) \Delta h \\
& -\int_{Q_{\tilde{T}}}\left(b(u)-b\left(u_{D}\right)\right) \nabla h \cdot \nabla \varphi\left(u_{D}\right) .
\end{aligned}
$$

The first term is estimated as

$$
\begin{aligned}
\int_{Q_{\tilde{T}}}\left(B(u)-B\left(u_{D}\right)\right) \Delta h & \leq\left\|B(u)-B\left(u_{D}\right)\right\|_{L^{p^{\prime}}}\|\Delta h\|_{L^{p}} \\
& \leq c\left(\left|Q_{\tilde{T}}\right|^{1 / p^{\prime}}+\|u\|_{L^{2 p^{\prime}}}^{2}+\left\|u_{D}\right\|_{L^{2 p^{\prime}}}^{2}\right)\|\Delta h\|_{L^{p}} .
\end{aligned}
$$

Since $h \in \tilde{K}$, and $2 p^{\prime} \leq r$ due to the choice of $p$, see $(2.11)$, we deduce $\|u\|_{L^{2 p^{\prime}}} \leq$ $c\|u\|_{L^{r}} \leq c\|\| u \mid \|$ and therefore

$$
\int_{Q_{\tilde{T}}}\left(B(u)-B\left(u_{D}\right)\right) \Delta h \leq c\left(\left|Q_{\tilde{T}}\right|^{1 / p^{\prime}}+\left\|u_{D}\right\|_{L^{2 p^{\prime}}}^{2}+\||u|\|^{2}\right) \rho .
$$

The second term is estimated as follows:

$$
\int_{Q_{\tilde{T}}}\left(b(u)-b\left(u_{D}\right)\right) \nabla h \cdot \nabla \varphi\left(u_{D}\right) \leq\left\|b(u)-b\left(u_{D}\right)\right\|_{L^{r}\left(L^{2}\right)}\|\nabla h\|_{L^{p}\left(L^{\infty}\right)}\left\|\nabla \varphi\left(u_{D}\right)\right\|_{L^{2}} .
$$

Since $p>N$ we have $\|\nabla h\|_{L^{p}\left(L^{\infty}\right)} \leq c\|\Delta h\|_{L^{\infty}}$. Using $b$ sublinear, $r>2$ and (2.10)(2.11) we obtain

$$
\begin{aligned}
\int_{Q_{\tilde{T}}}\left(b(u)-b\left(u_{D}\right)\right) \nabla h \cdot \nabla \varphi\left(u_{D}\right) \leq & c\left\|\nabla \varphi\left(u_{D}\right)\right\|_{L^{2}} \\
& \times\left(\left|Q_{\tilde{T}}\right|^{1 / p^{\prime}}+\left\|u_{D}\right\|_{L^{r}}+\||u|\|\right) \rho .
\end{aligned}
$$

We may obtain similar estimates from $v$-equation. Using $\varphi^{-1}$ Lipschitz continuous as in (2.19) we get from (2.36)-(2.38)

$$
\||u|\|^{2}+\||v|\|^{2} \leq \Lambda_{1}(\rho)+c_{3}\left(\||u|\|^{2}+\||v|\|^{2}\right)\left(\rho+\rho^{2}\right)
$$


with $\Lambda_{1}(\rho):=c_{1}+c_{2} \rho$, and $c_{1}, c_{2}, c_{3}$ depending on the norms of the auxiliary conditions and on $\tilde{T}^{\beta}$, for some $\beta>0$. Hence, defining $G(\rho, \tilde{T}):=\frac{\Lambda_{1}(\rho)}{\sqrt{1-c_{3}\left(\rho+\rho^{2}\right)}}$ with $\rho \in\left(0, c_{\rho}\right)$ and $c_{\rho}:=\min \left\{1, \frac{1}{2 c}\right\}$ we finish.

Now we can afford the

Proof of Theorem 2.1. The proof uses a regularization technique and Theorem 2.2. In view of the constructive method that we shall use in one of the uniqueness results, we consider two different regularizations of problem (1.1) depending on whether $\varphi$ is strictly increasing or only non-decreasing. In the first case we consider the following perturbation of the auxiliary data

$$
\begin{cases}\varphi\left(u_{D \varepsilon}\right)=\varphi\left(u_{D}\right)+\varphi\left(\varepsilon e^{-\lambda_{1} t}\right) & \text { on } \Sigma_{D} \\ \varphi\left(v_{D \varepsilon}\right)=\varphi\left(v_{D}\right)+\varphi\left(\varepsilon e^{-\lambda_{1} t}\right) & \text { on } \Sigma_{D} \\ u_{0 \varepsilon}=u_{0}+\varepsilon, \quad v_{0 \varepsilon}=v_{0}+\varepsilon & \text { in } \Omega\end{cases}
$$

for some $\lambda_{1}>0$, remaining the other auxiliary conditions the same, and we consider

$$
\varphi_{\varepsilon}(s):= \begin{cases}\varphi\left(\varepsilon e^{-\lambda T}\right) \exp \left\{\mu\left(s-\varepsilon e^{-\lambda T}\right)\right\} & \text { si } s<\varepsilon e^{-\lambda T}, \\ \varphi(s) & \text { si } s \in\left[\varepsilon e^{-\lambda T}, k\right], \\ \varphi^{\prime}(k) s+\varphi(k)-k \varphi^{\prime}(k) & \text { si } s \geq k,\end{cases}
$$

with $k$ an $L^{\infty}$ constant bound of the auxiliary data and $\mu:=\frac{\varphi^{\prime}\left(\varepsilon e^{-\lambda T}\right)}{\varphi\left(\varepsilon e^{-\lambda T}\right)}$. We have $\varphi_{\varepsilon} \in \mathcal{C}^{1}([0, \infty)), \varphi_{\varepsilon}(0)>0$ and $\varphi_{\varepsilon}^{\prime}>0$. It is straightforward to check that the sequence of problems $(1.1)_{\varepsilon}$ corresponding to (2.39) and (2.40) satisfy the conditions of Theorem 2.2. Finally, notice that $\varphi$ and $\varphi_{\varepsilon}$ coincides in the range of $u_{\varepsilon}, v_{\varepsilon}$.

In the case in which $\varphi$ is non-decreasing we consider, for each $\varepsilon>0$, the regularization given by $\varphi_{\varepsilon}(s):=\varphi(s)+\varepsilon s$ and leave the auxiliary conditions unchanged. It is easy to see that the requirements of Theorem 2.2 are satisfied, obtaining therefore the existence of a sequence of solutions of $(1.1)_{\varepsilon}$ with the regularity and properties stated in that theorem.

A priori estimates. In both cases we proceed in a similar way: we use $\varphi_{\varepsilon}\left(u_{\varepsilon}\right)-\varphi\left(u_{D \varepsilon}\right)$ as a test function for the first equation in (1.1) and as in step $\mathbf{7}$ of the proof of Theorem 2.2 , we obtain

$$
\sup _{0 \leq t \leq T} \int_{\Omega} \Phi\left(u_{\varepsilon}(t)\right)+\int_{Q_{T}}\left|\nabla \varphi\left(u_{\varepsilon}\right)\right|^{2}+\varepsilon \int_{Q_{T}}\left|\nabla u_{\varepsilon}\right|^{2} \leq C,
$$

with $C$ independent of $\varepsilon$ (because the $L^{\infty}$ bounds of $u_{\varepsilon}, v_{\varepsilon}$ are independent of $\varphi_{\varepsilon}$ ). Using now $\xi \in L^{2}(0, T ; \mathcal{V})$ as a test function we get

$$
\begin{gathered}
\left|\int_{0}^{T}\left\langle u_{\varepsilon t}, \xi\right\rangle_{\mathcal{V}^{\prime}, \mathcal{V}}\right| \leq\left\|\nabla \varphi_{\varepsilon}\left(u_{\varepsilon}\right)\right\|_{L^{2}}\|\nabla \xi\|_{L^{2}}+\left\|b\left(u_{\varepsilon}\right)\right\|_{L^{\infty}}\left\|\nabla w_{\varepsilon}\right\|_{L^{2}}\|\nabla \xi\|_{L^{2}} \\
+\left\|F\left(u_{\varepsilon}, v_{\varepsilon}\right)\right\|_{L^{2}}\|\xi\|_{L^{2}},
\end{gathered}
$$

from where we deduce

$$
\left\|u_{\varepsilon t}\right\|_{L^{2}\left(0, T ; \mathcal{V}^{\prime}\right)} \leq c,
$$

with $c$ independent of $\varepsilon$. A similar estimate holds for $v_{\varepsilon}$. From the third equation of (1.1) we get

$$
\left\|\Delta w_{\varepsilon}\right\|_{L^{\infty}\left(Q_{T}\right)} \leq\left\|v_{\varepsilon}-u_{\varepsilon}+C\right\|_{L^{\infty}\left(Q_{T}\right)} \leq \text { const. }
$$


Therefore, using estimates (2.41)-(2.43) and standard compactness results we can extract subsequences (labeled again by $\varepsilon$ ) such that

(2.44) $\begin{cases}u_{\varepsilon} \rightarrow u & \text { weakly } * \text { in } L^{\infty}\left(Q_{T}\right), \\ \varphi\left(u_{\varepsilon}\right) \rightarrow \xi & \text { weakly in } \varphi\left(u_{D}\right)+L^{2}(0, T ; \mathcal{V}), \\ u_{\varepsilon t} \rightarrow u_{t} & \text { weakly in } L^{2}\left(0, T ; \mathcal{V}^{\prime}\right), \\ \varepsilon^{1 / 2} u_{\varepsilon} \rightarrow 0 & \text { weakly in } L^{2}(0, T ; \mathcal{V}), \\ w_{\varepsilon} \rightarrow w & \text { weakly } * \text { - weakly in } L^{\infty}\left(0, T ; W^{2, s}(\Omega)\right) \text {, for all } s<\infty .\end{cases}$

From the compact imbedding $L^{\infty}(\Omega) \subset H^{-1}(\Omega)$ and Corollary 4 (p. 85) of [39] we obtain

$$
u_{\varepsilon} \rightarrow u \quad \text { in } \quad \mathcal{C}\left([0, T], \mathcal{V}^{\prime}\right) .
$$

Since $\varphi$ is continuous and non-decreasing we have that $-\Delta \varphi(\cdot)$ is a maximal monotone graph in $L^{2}\left(0, T ; \mathcal{V}^{\prime}\right)$ and, therefore, it is strongly-weakly closed in such space (see, e.g. [8]), from where we deduce

$$
\xi=\varphi(u) .
$$

Assume, now, $\mathbf{H}_{4}$. In order to pass to the limit on $b\left(u_{\varepsilon}\right)$ and $F\left(u_{\varepsilon}, v_{\varepsilon}\right)$ we shall prove that $u_{\varepsilon} \rightarrow u$ strongly in $L^{q}\left(Q_{T}\right)$ for all $q<\infty$. To do this we use a modification of the arguments given in [17], [31] or [19]. Defining the space

$$
\mathcal{H}=\left\{u \in L^{2 / \alpha}\left(0, T ; W^{\alpha, 2 / \alpha}(\Omega)\right), u_{t} \in L^{2}\left(0, T ; \mathcal{V}^{\prime}\right)\right\} .
$$

By (2.41) and (2.42) we have that $u_{\varepsilon}$ is uniformly bounded in $\mathcal{H}$. Then, from the compact imbedding $\mathcal{H} \subset L^{2 / \alpha}\left(Q_{T}\right)$ we deduce the existence of a subsequence of $u_{\varepsilon}$ such that

$$
u_{\varepsilon} \rightarrow u \text { strongly in } L^{2 / \alpha}\left(Q_{T}\right) \text { and a.e. in } Q_{T} .
$$

This fact together with the weak $*$ convergence of $u_{\varepsilon}$ to $u$ in $L^{\infty}\left(Q_{T}\right)$ implies that $u_{\varepsilon} \rightarrow u$ strongly in $L^{q}\left(Q_{T}\right)$ for all $q<\infty$. And similarly for $v$.

Identification of the limit. Let $\zeta \in L^{2}(0, T ; \mathcal{V})$ be a test function. By $(2.44)$ and $(2.45)$ it is clear that

$$
\int_{0}^{T}\left\langle u_{\varepsilon t}, \zeta\right\rangle \rightarrow \int_{0}^{T}\left\langle u_{t}, \zeta\right\rangle \quad \text { and } \quad \int_{0}^{T} \int_{\Omega} \nabla \varphi\left(u_{\varepsilon}\right) \cdot \nabla \zeta \rightarrow \int_{0}^{T} \int_{\Omega} \nabla \varphi(u) \cdot \nabla \zeta .
$$

From the pointwise convergence of $u_{\varepsilon}, v_{\varepsilon}$ to $u, v$ in $Q_{T}$, we get $F\left(u_{\varepsilon}, v_{\varepsilon}\right) \rightarrow F(u, v)$ a.e. in $Q_{T}$, and since $F$ is Lipschitz continuous we obtain

$$
\left\|F\left(u_{\varepsilon}, v_{\varepsilon}\right)\right\|_{L^{2}} \leq c\left(\left\|u_{\varepsilon}\right\|_{L^{2}}+\left\|v_{\varepsilon}\right\|_{L^{2}}+1\right) \leq \text { const. },
$$

so there exists $\tilde{F} \in L^{2}\left(Q_{T}\right)$ such that $F\left(u_{\varepsilon}, v_{\varepsilon}\right) \rightarrow \tilde{F}$ weakly in $L^{2}\left(Q_{T}\right)$. Lebesgue's theorem implies $\tilde{F} \equiv F(u, v)$, and therefore

$$
\int_{0}^{T} \int_{\Omega} F\left(u_{\varepsilon}, v_{\varepsilon}\right) \zeta \rightarrow \int_{0}^{T} \int_{\Omega} F(u, v) \zeta
$$

Similarly we obtain $b\left(u_{\varepsilon}\right) \rightarrow b(u)$ and $b\left(v_{\varepsilon}\right) \rightarrow b(v)$ strongly in $L^{q}\left(Q_{T}\right)$ for all $q<\infty$. Due to the compact imbedding $L^{2}\left(0, T ; W^{2,2}(\Omega)\right) \subset L^{2}(0, T ; \mathcal{V})$ we also have $w_{\varepsilon} \rightarrow w$ strongly in $L^{2}(0, T ; \mathcal{V})$. We, finally, deduce

$$
\int_{Q_{T}} b\left(u_{\varepsilon}\right) \nabla w_{\varepsilon} \cdot \nabla \zeta \rightarrow \int_{Q_{T}} b(u) \nabla w \cdot \nabla \zeta .
$$


So the limit, $u$, has been identified as the first component of a solution of (1.1). The other components are handled in a very similar way and we skip therefore the proof. In the case in which $\mathbf{H}_{4}$ does not hold, i.e., when both $b$ and $F$ are linear functions, the passing to the limit is easier because we do not need to ensure the pointwise convergence of $u_{\varepsilon}, v_{\varepsilon}$ to $u, v$. In this situation the identification of the limit is just a consequence of the weak convergences in (2.44) and (2.45). Finally, from [3], Theorem 2.2 , we obtain the additional regularity

$$
\sqrt{\varphi^{\prime}(u)} \nabla u, \sqrt{\varphi^{\prime}(v)} \nabla v \in L^{2}\left(Q_{T}\right)
$$

To finish, notice that due to Theorem 2.2 we have that, for all $\varepsilon>0,(2.7)$ holds. We then deduce that this property also holds in the limit $\varepsilon \rightarrow 0$. $\square$

The following regularity result will be used in the uniqueness section, see Theorem 3.4 .

Corollary 2.3. In the conditions of Theorem 2.1, let $\varphi \in \mathcal{C}^{1}([0, \infty))$ be strictly increasing with $\varphi^{\prime}(0)=0$. Assume

$$
\int_{0}^{s} \frac{b^{\prime}(\sigma)^{2}}{\varphi^{\prime}(\sigma)} d \sigma<\infty \quad \text { and } \quad \int_{0}^{s} \frac{b(\sigma) b^{\prime}(\sigma)^{2}}{\varphi^{\prime}(\sigma)} d \sigma<\infty \quad \text { for all } s \in[0, \infty) .
$$

Then there exists a solution of problem (1.1) with the regularity given in Theorem 2.1 and

$$
b(u), b(v) \in L^{2}(0, T ; \mathcal{V})
$$

Remark. Let $\varphi(s):=s^{m}$ and $b(s):=s^{\gamma}$ with $m>1, \gamma \geq 1$. If $m<2 \gamma$ then (2.47) is satisfied.

Proof. We consider the sequence of solutions $\left(u_{\varepsilon}, v_{\varepsilon}, w_{\varepsilon}\right)$ of problems $(1.1)_{\varepsilon}$ constructed as in proof of Theorem 2.1. Since $u_{\varepsilon} \geq c \varepsilon$ we have that $\psi\left(u_{\varepsilon}\right)-\psi\left(u_{D \varepsilon}\right)$ is an admissible test function for any $\varepsilon>0$. For simplicity, we suppose $u_{D}=0$ in $\Sigma_{D}$ and therefore $u_{D \varepsilon}=\varepsilon e^{-\lambda_{1} t}$. We have for the diffusion term

$$
\int_{Q_{T}} \nabla \varphi\left(u_{\varepsilon}\right) \cdot \nabla\left(\psi\left(u_{\varepsilon}\right)-\psi\left(u_{D \varepsilon}\right)\right)=\int_{Q_{T}} \frac{\varphi^{\prime}\left(u_{\varepsilon}\right) \psi^{\prime}\left(u_{\varepsilon}\right)}{b^{\prime}\left(u_{\varepsilon}\right)^{2}}\left|\nabla b\left(u_{\varepsilon}\right)\right|^{2}=\int_{Q_{T}}\left|\nabla b\left(u_{\varepsilon}\right)\right|^{2},
$$

with $\psi(s)=\int_{0}^{s} b^{\prime}(\sigma)^{2} / \varphi^{\prime}(\sigma) d \sigma$. For the drift term we get

$$
\begin{aligned}
\int_{Q_{T}} b\left(u_{\varepsilon}\right) \nabla w_{\varepsilon} \cdot \nabla\left(\psi\left(u_{\varepsilon}\right)-\psi\left(u_{D \varepsilon}\right)\right)= & \int_{Q_{T}} \nabla w_{\varepsilon} \cdot \nabla B\left(u_{\varepsilon}\right)=\int_{Q_{T}}\left(v_{\varepsilon}-u_{\varepsilon}+C\right) B\left(u_{\varepsilon}\right) \\
& +\int_{\Sigma_{D}} B\left(u_{D \varepsilon}\right) \nabla w_{\varepsilon} \cdot \nu
\end{aligned}
$$

with $B(s):=\int_{0}^{s} b(\sigma) \psi^{\prime}(\sigma) d \sigma$. For the time derivative term we obtain

$$
\begin{aligned}
\int_{Q_{T}} u_{\varepsilon t}\left(\psi\left(u_{\varepsilon}\right)-\psi\left(u_{D \varepsilon}\right)\right)= & \varepsilon \lambda_{1} \int_{Q_{T}} u_{\varepsilon} \psi^{\prime}\left(u_{D \varepsilon}\right)+\int_{\Omega}\left(G\left(u_{\varepsilon}(T)\right)-G\left(u_{\varepsilon 0}\right)\right) \\
& -\int_{\Omega}\left(u_{\varepsilon}(T) \psi\left(u_{D \varepsilon}(T)\right)-u_{\varepsilon 0} \psi\left(u_{D \varepsilon}(0)\right)\right)
\end{aligned}
$$


with $G(s):=\int_{0}^{s} \psi(\sigma) d \sigma$. We have then

$$
\begin{aligned}
\int_{Q_{T}}\left|\nabla b\left(u_{\varepsilon}\right)\right|^{2} \leq & \int_{\Omega} G\left(u_{\varepsilon 0}\right)+u_{\varepsilon}(T) \psi\left(u_{D \varepsilon}(T)\right)+\int_{Q_{T}}\left(v_{\varepsilon}-u_{\varepsilon}+C\right) B\left(u_{\varepsilon}\right) \\
& +\int_{\Sigma_{D}} B\left(u_{D \varepsilon}\right) \nabla w_{\varepsilon} \cdot \nu+\int_{Q_{T}} F\left(u_{\varepsilon}, v_{\varepsilon}\right)\left(\psi\left(u_{\varepsilon}\right)-\psi\left(u_{D \varepsilon}\right)\right)
\end{aligned}
$$

Using the $L^{\infty}\left(Q_{T}\right)$ uniform bounds of $u_{\varepsilon}, v_{\varepsilon}$ and property $(2.47)$ we deduce

$$
\int_{Q_{T}}\left|\nabla b\left(u_{\varepsilon}\right)\right|^{2} \leq c \quad \text { for all } \varepsilon>0 .
$$

We find then by $(2.44)$ that $b\left(u_{\varepsilon}\right) \rightarrow b(u)$ weakly in $L^{2}(0, T ; \mathcal{V})$, with $u$ the first component of a solution of (1.1). We may follow the same argument to deduce the property for $v$.

Remark. The technique we have used is also applicable when $F(u, v)$ is a maximal monotone graph, see [15] for a likely system but without transport terms. We also point out that functions $\varphi(u)$ and $\varphi(v)$ as well as $b(u)$ and $b(v)$ may be different as long as they fulfill the assumptions given on the data.

3. Uniqueness of solutions. We present in this section three theorems on the uniqueness question for problem (1.1) which share a duality technique in their proofs, i.e., the searching of suitable test functions which allow to deduce the uniqueness property.

The first result is obtained by using a technique introduced by Antontsev, Díaz and Domansky [2] for a system of two-phase filtration in porous medium. Here we assume $\left(b^{\prime}(s)\right)^{2} \leq c \varphi^{\prime}(s)$, which holds in the case when diffusion and transport are both linear or in the case in which they are degenerate in a suitable way. It is worth noting that this type of condition also arises as sufficient condition to ensure the existence of strong solutions of (1.2), see [6].

The second result uses a technique introduced by Rulla [37] to study the Stefan problem with prescribed convection. In this case we only assume $\varphi$ non-decreasing, but an entropy type condition for the electric field on the Dirichlet boundary must be introduced: $\nabla w \cdot \nu=0$ on $\Sigma_{D}$. Conditions of this type are already classical in the literature of hyperbolic equations, see [28], and they arise as natural conditions which allow to select a unique solution (the so-called entropy solution) when uniqueness fails for weak solutions.

Our last theorem applies to the case in which problem (1.1) has strong solutions in the following sense: $b(u), b(v) \in L^{1}(0, T ; \mathcal{W})$, with

$$
\mathcal{W}:=\left\{h \in W^{1, p}(\Omega): h=0 \text { on } \Gamma_{D}\right\},
$$

and with $p>N$ if $N \geq 2$ and $p=1$ if $N=1$. To obtain this result we used a method due to Kalashnikov [26] which consists of making a comparaison between an arbitrary weak solution of (1.1) and the weak solution constructed as the limit of a sequence of solutions of regularized problems, see proof of Theorem 2.1. Our result is strongly based on the technique introduced by Díaz and Kersner [13] to study a one dimensional scalar equation.

In the sequel we shall assume that the component $w$ of solutions is non-trivial in the sense that $\|\nabla w\|_{L^{2}\left(Q_{T}\right)} \neq 0$. On the contrary, the system reduces to the equation 
$u_{t}-\Delta \varphi(u)=F(u, u-C)$, in fact simpler than (1.2) whichis , as we already mentioned, well understood.

THEOREM 3.1. Assume $\mathbf{H}_{1}-\mathbf{H}_{3}$ and suppose that there exists a constant $M>0$ such that

$$
\left(b^{\prime}(s)\right)^{2} \leq M \varphi^{\prime}(s) \text { for all } s>0
$$

and

$$
\left(\frac{\partial}{\partial s_{i}} F\left(s_{1}, s_{2}\right)\right)^{2} \leq M \varphi^{\prime}\left(s_{i}\right), \quad \text { for all } s_{i}>0, \quad i=1,2
$$

Then problem (1.1) has a unique weak solution.

Proof. Suppose that $\left(u_{1}, v_{1}, w_{1}\right)$ and $\left(u_{2}, v_{2}, w_{2}\right)$ are two weak solutions of (1.1) and define $(u, v, w):=\left(u_{1}-u_{2}, v_{1}-v_{2}, w_{1}-w_{2}\right), F_{i}:=F\left(u_{i}, v_{i}\right), i=1,2$ and $\hat{F}:=F_{1}-F_{2}$. Then $(u, v, w)$ satisfies

$$
\left\{\begin{array}{l}
u_{t}-\Delta\left(\varphi\left(u_{1}\right)-\varphi\left(u_{2}\right)\right)+\operatorname{div}\left(b\left(u_{1}\right) \nabla w+\left(b\left(u_{1}\right)-b\left(u_{2}\right)\right) \nabla w_{2}\right)=\hat{F} \\
v_{t}-\Delta\left(\varphi\left(v_{1}\right)-\varphi\left(v_{2}\right)\right)-\operatorname{div}\left(b\left(v_{1}\right) \nabla w+\left(b\left(v_{1}\right)-b\left(v_{2}\right)\right) \nabla w_{2}\right)=\hat{F} \\
-\Delta w+u-v=0
\end{array}\right.
$$

in $Q_{T}$, with auxiliary conditions

$$
\left\{\begin{array}{lll}
\nabla \varphi\left(u_{i}\right) \cdot \nu=0, \quad \nabla \varphi\left(v_{i}\right) \cdot \nu=0, & \nabla w \cdot \nu=0 & \text { on } \Sigma_{N} \\
\varphi\left(u_{i}\right)=\varphi\left(u_{D}\right), \quad \varphi\left(v_{i}\right)=\varphi\left(v_{D}\right), & w=0 & \text { on } \Sigma_{D} \\
u(\cdot, 0)=0, \quad v(\cdot, 0)=0 & & \text { in } \Omega
\end{array}\right.
$$

$i=1,2$. Taking smooth test functions $\psi, \xi, \eta$ with homogeneous mixed boundary conditions for problem (3.4)-(3.5), integrating by parts and adding the resulting integral identities we obtain

$$
\begin{aligned}
\int_{\Omega} \psi(T) u(T)+\xi(T) v(T)= & \int_{Q_{T}} u\left(\psi_{t}+A_{u} \Delta \psi+\mathbf{B}_{u} \cdot \nabla \psi+\eta+F_{u}(\psi+\xi)\right) \\
& +\int_{Q_{T}} v\left(\xi_{t}+A_{v} \Delta \xi-\mathbf{B}_{v} \nabla \xi-\eta+F_{v}(\psi+\xi)\right) \\
& -\int_{Q_{T}} w \operatorname{div}\left(b\left(u_{1}\right) \nabla \psi-b\left(v_{1}\right) \nabla \xi+\nabla \eta\right),
\end{aligned}
$$

with $A_{z}:=\int_{0}^{1} \varphi^{\prime}\left(s z_{1}+(1-s) z_{2}\right) d s, \mathbf{B}_{z}:=\nabla w_{2} \int_{0}^{1} b^{\prime}\left(s z_{1}+(1-s) z_{2}\right) d s$ for $z=u, v$ and $F_{u}:=\int_{0}^{1} \frac{\partial F}{\partial u}\left(s u_{1}+(1-s) u_{2}, v_{1}\right) d s$ with a similar definition for $F_{v}$. Notice that since $b \in \mathcal{C}^{1}([0, \infty)), u_{i}, v_{i} \in L^{\infty}\left(Q_{T}\right), F$ is Lipschitz continuous and $\nabla w_{2} \in L^{\infty}\left(Q_{T}\right)$ we have that $\mathbf{B}_{z}, F_{z}$ are bounded in $L^{\infty}\left(Q_{T}\right)$, for $z=u, v$. We define the differential operators

$$
\begin{aligned}
& \mathcal{L}_{1}(\psi, \xi, \eta):=\psi_{t}+A_{u}^{\varepsilon} \Delta \psi+\mathbf{B}_{u} \cdot \nabla \psi+\eta+F_{u}(\psi+\xi) \\
& \mathcal{L}_{2}(\psi, \xi, \eta):=\xi_{t}+A_{v}^{\varepsilon} \Delta \xi-\mathbf{B}_{v} \cdot \nabla \xi-\eta+F_{v}(\psi+\xi) \\
& \mathcal{L}_{3}(\psi, \xi, \eta):=\operatorname{div}\left(b\left(u_{1}\right) \nabla \psi-b\left(v_{1}\right) \nabla \xi+\nabla \eta\right)
\end{aligned}
$$

with $A_{u}^{\varepsilon}:=A_{u}+\varepsilon$ and $\varepsilon>0$, (and a similar definition for $A_{v}^{\varepsilon}$ ) and set the following 
problem to choose the test functions:

$$
\begin{cases}\mathcal{L}_{1}(\psi, \xi, \eta)=u & \text { in } Q_{T}, \\ \mathcal{L}_{2}(\psi, \xi, \eta)=v & \text { in } Q_{T}, \\ \mathcal{L}_{3}(\psi, \xi, \eta)=0 & \text { in } Q_{T}, \\ \nabla \psi \cdot \nu=\nabla \xi \cdot \nu=\nabla \eta \cdot \nu=0 & \text { on } \Sigma_{N}, \\ \psi=\xi=\eta=0 & \text { on } \Sigma_{D}, \\ \psi(T)=\xi(T)=0 & \text { in } \Omega .\end{cases}
$$

LEMma 3.2. Problem (3.7) has a unique solution with the regularity of test functions of (1.1), see (2.4) and (2.5). Moreover,

$$
\psi, \xi, \eta \in H^{1}\left(0, T ; L^{2}(\Omega)\right) \cap L^{\infty}\left(0, T ; H^{1}(\Omega)\right) \cap L^{2}\left(0, T ; H^{2}(\Omega)\right),
$$

and there exists a positive constant $C(T)$ independent of $\varepsilon$ such that

$$
\varepsilon \int_{Q_{T}}\left(|\Delta \psi|^{2}+|\Delta \xi|^{2}\right) \leq C(T) .
$$

Continuation of proof of Theorem 3.1. Introducing in (3.6) the test functions provided by Lemma 3.2 we get

$$
\int_{Q_{T}}\left(u^{2}+v^{2}\right)=\varepsilon \int_{Q_{T}}(u \Delta \psi+v \Delta \xi) .
$$

Young's inequality and (3.8) leads to

$$
\int_{Q_{T}}\left(u^{2}+v^{2}\right) \leq \sqrt{\varepsilon}\left(\int_{Q_{T}}\left(u^{2}+v^{2}\right)+C(T)\right) .
$$

Hence, taking the limit $\varepsilon \rightarrow 0$, we conclude that $u \equiv v \equiv 0$ a.e. in $Q_{T}$, which also implies $w \equiv 0$ a.e. in $Q_{T}$.

Proof of Lemma 3.2.

Step 1. A prori estimates. Multiplying the third equation of (3.7) by $\eta$ and using the regularity $u_{i}, v_{i} \in L^{\infty}\left(Q_{T}\right)$ and the continuity of $b$ we get

$$
\int_{\Omega}|\nabla \eta|^{2} \leq c_{0}(T) \int_{\Omega}\left(|\nabla \psi|^{2}+|\nabla \xi|^{2}\right)
$$

with $c_{0}(T) \geq 0$. Thanks to $(3.2)$ we can estimate

$$
\int_{\Omega}\left(\mathbf{B}_{u} \cdot \nabla \psi\right) \Delta \psi \leq \delta \int_{\Omega} A_{u}^{\varepsilon}|\Delta \psi|^{2}+\frac{M}{\delta} \int_{\Omega}|\nabla \psi|^{2},
$$

for $\delta>0$. A similar estimate holds, due to (3.3), for $\int_{\Omega} F_{u}(\psi+\xi) \Delta \psi$. Multiplying the first equation of (3.7) by $\Delta \psi$ and using (3.10), the analogous expression for the $F$ term we obtain, for a suitable $\delta$

$$
\begin{aligned}
-\frac{1}{2} \frac{d}{d t} \int_{\Omega}|\nabla \psi|^{2}+\int_{\Omega} A_{u}^{\varepsilon}|\Delta \psi|^{2} \leq & c\left(\int_{\Omega}\left(|\nabla \psi|^{2}+|\nabla \xi|^{2}\right)\right. \\
& \left.+\int_{\Omega}|\nabla \eta|^{2}+\int_{Q_{T}} u^{2}\right) .
\end{aligned}
$$


From the second equation of (3.7) we obtain a similar inequality for $\xi$ which, being added to (3.11) and taking into account that $A_{u}^{\varepsilon}, A_{v}^{\varepsilon}>\varepsilon$ and (3.9) allows us to deduce

$$
\begin{aligned}
-\frac{1}{2} \frac{d}{d t} \int_{\Omega}\left(|\nabla \psi|^{2}+|\nabla \xi|^{2}\right)+\frac{\varepsilon}{2} \int_{\Omega}\left(|\Delta \psi|^{2}+|\Delta \xi|^{2}\right) \leq & c\left(\int_{\Omega}\left(|\nabla \psi|^{2}+|\nabla \xi|^{2}\right)\right. \\
& \left.+\int_{\Omega}\left(u^{2}+v^{2}\right)\right)
\end{aligned}
$$

with $c(T)$ independent of $\varepsilon$. On one hand, we deduce from Gronwall's Lemma that

$$
\int_{\Omega}\left(|\nabla \psi(t)|^{2}+|\nabla \xi(t)|^{2}\right) \leq c_{1}(T) e^{T c(T)},
$$

with $c_{1}(T)$ independent of $\varepsilon$. Note that both $c$ and $c_{1}$ are increasing and uniformly continuous functions of $T$. They just depend on norms of the data. On the other hand, integrating (3.13) in $(0, T)$ and using (3.14) we obtain

$$
\frac{\varepsilon}{2} \int_{Q_{T}}\left(|\Delta \psi|^{2}+|\Delta \xi|^{2}\right) \leq c_{2}(T) e^{T c(T)},
$$

with $c_{2}(T)$ independent of $\varepsilon$. So we deduced (3.8). Finally, from the third equation of (3.7) we have that

$$
\Delta \eta=\nabla b\left(v_{1}\right) \cdot \nabla \xi+b\left(v_{1}\right) \Delta \xi-\nabla b\left(u_{1}\right) \cdot \nabla \psi-b\left(u_{1}\right) \Delta \psi
$$

and from (3.2) and the regularity $\sqrt{\varphi^{\prime}\left(v_{1}\right)} \nabla v_{1} \in L^{2}\left(Q_{T}\right)$, see Theorem 2.1, we obtain

$$
\int_{\Omega}\left|\nabla b\left(v_{1}\right)\right|^{2}=\int_{\Omega} b^{\prime}\left(v_{1}\right)^{2}\left|\nabla v_{1}\right|^{2} \leq c \int_{\Omega} \varphi^{\prime}\left(v_{1}\right)\left|\nabla v_{1}\right|^{2} \leq \text { const. }
$$

Hence, using Hölder and Young's inequalities and estimates (3.9) and (3.16) we obtain the $L^{2}\left(0, T ; H^{2}(\Omega)\right.$ regularity of $\eta$.

Step 2. Existence of solutions of (3.7). We use fixed point argument. Consider the set

$$
K:=\left\{h \in L^{2}\left(0, T^{*} ; \mathcal{V}\right):\|h\|_{L^{2}\left(0, T^{*} ; \mathcal{V}\right)} \leq R\right\}
$$

where $T^{*}$ and $R$ will be suitably chosen. $K$ is convex and weakly compact in $L^{2}\left(0, T^{*} ; \mathcal{V}\right)$. We define $Q: K \subset L^{2}\left(0, T^{*} ; \mathcal{V}\right) \rightarrow L^{2}\left(0, T^{*} ; \mathcal{V}\right)$ by $Q(\hat{\eta}):=\eta$, where $\eta$ is the unique solution of $\mathcal{L}_{3}(\hat{\psi}, \hat{\xi}, \eta)=0$, being $(\hat{\psi}, \hat{\xi})$ the unique solution of

$$
\left\{\begin{array}{l}
\mathcal{L}_{1}(\hat{\psi}, \hat{\xi}, \hat{\eta})=u \\
\mathcal{L}_{2}(\hat{\psi}, \hat{\xi}, \hat{\eta})=v
\end{array}\right.
$$

with the same auxiliary conditions as in (3.7). Since $u, v, \nabla \hat{\eta} \in L^{2}\left(Q_{T^{*}}\right)$ we can justify the a priori estimates in Step 1 and deduce that any solution of (3.17) satisfies

$$
\hat{\psi}, \hat{\xi} \in H^{1}\left(0, T^{*} ; L^{2}(\Omega)\right) \cap L^{\infty}\left(0, T^{*} ; H^{1}(\Omega)\right) \cap L^{2}\left(0, T^{*} ; H^{2}(\Omega)\right) .
$$

Uniqueness of solutions of (3.17) follows from (3.18) and linearity of the differential operators. Existence of solutions of (3.17) is proven by uncoupling the problem and applying again a fixed point technique. Assume for the moment that such a solution 
exists and, therefore, it is unique and satisfies (3.18). We easily deduce that the solution of $\mathcal{L}_{3}(\hat{\psi}, \hat{\xi}, \eta)=0$ satisfies $\eta \in L^{2}\left(0, T^{*} ; H^{2}(\Omega)\right)$. Notice that if $\hat{\eta}$ is a fixed point of $Q$ then $(\hat{\psi}, \hat{\xi}, \hat{\eta})$ is a local solution of (3.7). To prove the existence of such a fixed point we shall show

(i) $Q(K) \subset K$, for suitable $R, T^{*}>0$,

(ii) $Q$ is weakly-weakly sequentially continuous in $L^{2}\left(0, T^{*} ; \mathcal{V}\right)$,

and apply the fixed point theorem [4]. (i) follows from the previous a priori estimates. From (3.9) we find

$$
\|\eta\|_{L^{2}\left(0, T^{*} ; \mathcal{V}\right)} \leq c_{0}\left(T^{*}\right)\left(\|\hat{\psi}\|_{L^{2}\left(0, T^{*} ; \mathcal{V}\right)}+\|\hat{\xi}\|_{L^{2}\left(0, T^{*} ; \mathcal{V}\right)}\right),
$$

and from (3.11) and the corresponding estimate for $\xi$ we obtain

$$
\|\hat{\psi}\|_{L^{2}\left(0, T^{*} ; \mathcal{V}\right)}+\|\hat{\xi}\|_{L^{2}\left(0, T^{*} ; \mathcal{V}\right)} \leq c_{1}\left(T^{*}\right)\|\hat{\eta}\|_{L^{2}\left(0, T^{*} ; \mathcal{V}\right)} e^{c T^{*}}
$$

It follows that

$$
\|Q(\hat{\eta})\|_{L^{2}\left(0, T^{*} ; \mathcal{V}\right)} \leq c_{3}\left(T^{*}\right) e^{c T^{*}} R .
$$

Notice that, as we already mentioned, functions $c_{i}\left(T^{*}\right)$ are uniformly continuous and non.decreasing with $c_{i}(0)=0$ (they depend on the norms of the data in $Q_{T}$ ) and therefore we can take $T^{*}$ small enough to obtain $c_{3}\left(T^{*}\right) e^{c T^{*}} \leq 1$, deducing $Q(K) \subset K$. In fact, these properties of $c_{i}$ imply the global existence (once the local existence is proven). (ii) is a direct consequence of the linearity and regularity and we omit the proof. This finishes the proof of the existence of a fixed point and, therefore, of a local solution of (3.7). We already mentioned why, in fact, the solution is global. Finally, the uniqueness of solutions is again a consequence of the linearity of the problem and the regularity of the solution. To finish, notice that the proof of existence of solutions of (3.17) may be performed in a similar way.

Following, we state the second result on uniqueness of solutions of (1.1). The main feature of this theorem is that it allows to consider a nonlinear diffusion, $\varphi$, not necessarily strictly increasing. However, we need to assume that an entropy type condition on the electric field holds on the Dirichlet boundary.

Theorem 3.3. Assume $\mathbf{H}_{1}-\mathbf{H}_{3}$ and let $b(s)=s$. If

$$
\nabla w \cdot \nu=0 \quad \text { on } \quad \Gamma_{D} \times(0, T),
$$

and

$$
\left|F\left(s_{1}, \sigma_{1}\right)-F\left(s_{2}, \sigma_{2}\right)\right| \leq c\left[\left(\varphi\left(s_{1}\right)-\varphi\left(s_{2}\right)\right)+\left(\varphi\left(\sigma_{1}\right)-\varphi\left(\sigma_{2}\right)\right)\right]
$$

for all $s_{i}, \sigma_{i} \geq 0, i=1,2$, then problem (1.1) has a unique solution in the class of weak solutions such that

$$
w \in L^{\infty}\left(0, T ; W^{2, \infty}(\Omega)\right)
$$

Remark. The equality in (3.19) is a consequence of the different sign that transport terms have in $u$ and $v$-equations. Indeed, suppose that there exist two solutions 
$\left(u_{1}, \tilde{v}, w_{1}\right)$ and $\left(u_{2}, \tilde{v}, w_{2}\right)$. Then, under the conditions of Theorem 3.3, with the equality sign in (3.19) replaced by $\geq$, uniqueness follows.

Proof of Theorem 3.3. As in proof of Theorem 3.1 we obtain the following identity

$$
\begin{aligned}
\int_{Q_{T}} u_{t} \psi+v_{t} \xi= & \int_{Q_{T}}\left(\varphi\left(u_{1}\right)-\varphi\left(u_{2}\right)\right) \Delta \psi+u \nabla w_{2} \cdot \nabla \psi+u \eta \\
& +\int_{Q_{T}}\left(\varphi\left(v_{1}\right)-\varphi\left(v_{2}\right)\right) \Delta \xi-v \nabla w_{2} \cdot \nabla \xi-v \eta \\
& -\int_{Q_{T}} w \operatorname{div}\left(u_{1} \nabla \psi-v_{1} \nabla \xi+\nabla \eta\right)-\int_{Q_{T}}\left(F_{1}-F_{2}\right)(\psi+\xi) .
\end{aligned}
$$

We choose the test functions as solutions of the problem

$$
\begin{cases}-\Delta \psi(t)=u(t) & \text { in } \Omega, \\ -\Delta \xi(t)=v(t) & \text { in } \Omega, \\ -\Delta \eta(t)=\operatorname{div}\left(v_{1}(t) \nabla \xi(t)-u_{1}(t) \nabla \psi(t)\right) & \text { in } \Omega, \\ \nabla \psi \cdot \nu=\nabla \xi \cdot \nu=\nabla \eta \cdot \nu=0 & \text { on } \Sigma_{N} \\ \psi=\xi=\eta=0 & \text { on } \Sigma_{D}\end{cases}
$$

for a.e. $t \in(0, T)$. Existence, uniqueness and regularity of solutions is a consequence of the theory of linear elliptic equations. In particular, since $u, v \in \mathcal{C}\left([0, T] ; \mathcal{V}^{\prime}\right)$ we deduce $\psi, \xi \in \mathcal{C}([0, T] ; \mathcal{V})$. Then, $u(\cdot, 0)=v(\cdot, 0)=0$ in $\Omega$ and the homogeneous boundary conditions for $\psi$ and $\xi$ imply

$$
\nabla \psi(\cdot, 0)=\nabla \xi(\cdot, 0)=0 \quad \text { in } L^{2}(\Omega) .
$$

Using these test functions we get

$$
\begin{aligned}
\frac{1}{2} \int_{\Omega}\left(|\nabla \psi(T)|^{2}+|\nabla \xi(T)|^{2}\right)+ & \int_{Q_{T}}\left[u\left(\varphi\left(u_{1}\right)-\varphi\left(u_{2}\right)\right)+v\left(\varphi\left(v_{1}\right)-\varphi\left(v_{2}\right)\right)\right] \\
= & \int_{Q_{T}} \nabla w_{2} \cdot(u \nabla \psi-v \nabla \xi) \\
& +\int_{Q_{T}}\left[\nabla(\psi-\xi) \cdot \nabla \eta-\left(F_{1}-F_{2}\right)(\psi+\xi)\right] .
\end{aligned}
$$

Now we perform the arguments to handle the terms involving $u$. The terms involving $v$ are similarly treated after a change of sign. Due to the choice of the test functions

$$
\int_{Q_{T}} u \nabla w_{2} \cdot \nabla \psi=-\int_{Q_{T}} \Delta \psi \nabla w_{2} \cdot \nabla \psi
$$

As in [37], let us show that (3.19) implies

$$
-\int_{Q_{T}} \Delta \psi \nabla w_{2} \cdot \nabla \psi \leq \frac{1}{2}\left\|w_{2}\right\|_{L^{\infty}\left(W^{2, \infty}\right)} \int_{Q_{T}}|\nabla \psi|^{2} .
$$

Integrating formally by parts the left hand side of (3.24) we get

$(3.25) \int_{\Omega}-\Delta \psi\left(\nabla w_{2} \cdot \nabla \psi\right)=\int_{\Omega} \nabla \psi \cdot \nabla\left(\nabla w_{2} \cdot \nabla \psi\right)-\int_{\partial \Omega}\left(\nabla w_{2} \cdot \nabla \psi\right)(\nabla \psi \cdot \nu)$. 
See [37] for a rigorous derivation of this identity. Using that $\psi=0$ on $\Gamma_{D}$ implies that $\nabla \psi$ has the same direction as $\nu$ on $\Gamma_{D}$ we obtain

$$
\int_{\partial \Omega}\left(\nabla w_{2} \cdot \nabla \psi\right)(\nabla \psi \cdot \nu)=\int_{\Gamma_{D}}|\nabla \psi|^{2} \nabla w_{2} \cdot \nu .
$$

Denoting by $H(\cdot)$ the Hessian matrix we get after integrating by parts

$$
\begin{aligned}
\int_{\Omega} \nabla \psi \cdot \nabla\left(\nabla w_{2} \cdot \nabla \psi\right)= & \int_{\Omega} \nabla \psi: H\left(w_{2}\right): \nabla \psi-\frac{1}{2} \int_{\Omega} \Delta w_{2}|\nabla \psi|^{2} \\
& +\frac{1}{2} \int_{\Gamma_{D}}|\nabla \psi|^{2} \nabla w_{2} \cdot \nu
\end{aligned}
$$

Substituting (3.26) and (3.27) in (3.25) leads to

$$
\begin{aligned}
-\int_{\Omega} \Delta \psi\left(\nabla w_{2} \cdot \nabla \psi\right)= & \int_{\Omega} \nabla \psi: H\left(w_{2}\right): \nabla \psi-\frac{1}{2} \int_{\Omega} \Delta w_{2}|\nabla \psi|^{2} \\
& -\frac{1}{2} \int_{\Gamma_{D}}|\nabla \psi|^{2} \nabla w_{2} \cdot \nu
\end{aligned}
$$

and using $\nabla w_{2} \cdot \nu \geq 0$ on $\Gamma_{D}$ (as a particular case of (3.19)) and the regularity assumed on $w_{2}$ we deduce (3.24). For problem (3.21) estimate (3.9) holds and then we have

$$
\int_{Q_{T}}(\nabla \psi \cdot \nabla \eta-\nabla \xi \cdot \nabla \eta) \leq c \int_{Q_{T}}\left(|\nabla \psi|^{2}+|\nabla \xi|^{2}\right)
$$

Finally, Hölder's, Young's and Poincaré's inequalities together with (3.20) gives

$$
\begin{aligned}
-\int_{Q_{T}}\left(F_{1}-F_{2}\right)(\psi+\xi) \leq & \varepsilon c \int_{Q_{T}}\left[\left(\varphi\left(u_{1}\right)-\varphi\left(u_{2}\right)\right)^{2}+\left(\varphi\left(v_{1}\right)-\varphi\left(v_{2}\right)\right)^{2}\right] \\
& +\frac{c}{\varepsilon} \int_{Q_{T}}\left(|\nabla \psi|^{2}+|\nabla \xi|^{2}\right),
\end{aligned}
$$

for all $\varepsilon>0$. Then, using $\varphi$ Lipschitz continuous and non-decreasing, substituting estimates (3.24) (and the corresponding for $v),(3.28)$ and (3.29) in (3.23) and choosing $\varepsilon, \delta$ small enough we obtain

$$
\int_{\Omega}\left(|\nabla \psi(T)|^{2}+|\nabla \xi(T)|^{2}\right) \leq c \int_{Q_{T}}\left(|\nabla \psi|^{2}+|\nabla \xi|^{2}\right) .
$$

Gronwall's inequality and (3.22) imply $\nabla \psi \equiv \nabla \xi \equiv 0$ a.e. in $Q_{T}$, from where the assertion follows.

We finally present our third result. The main assumption is on the regularity of the solution constructed in Theorem 2.1: we suppose $\nabla b(u), \nabla b(v) \in L^{1}\left(0, T ; L^{p}(\Omega)\right)$, with $p$ given in (3.1). As a consequence of Corollary 2.3 this regularity property is satisified in the following example:

$$
\varphi(s):=s^{m}, \quad b(s):=s^{\gamma} \quad \text { with } 1<m<2 \gamma \text { and } \gamma \geq 1,
$$

in space dimension one.

THEOREM 3.4. Assume $\mathbf{H}_{1}-\mathbf{H}_{3}$ and suppose that there exists an open set $\tilde{B} \subset \Gamma_{D}$ such that the $(N-1)$-dimensional Haussdorf measure of $\tilde{B}$ and $\Gamma_{D}$ coincides. Suppose

$$
\varphi \in C^{2}((0, \infty)), \quad \text { with } \varphi^{\prime}(0)=0
$$


and assume the existence of a positive constant $c$ and a convex function $\mu \in C^{0}([0, \infty))$ $\cap C^{2}((0, \infty))$ such that $\mu(0)=0$,

$$
0<\mu^{\prime}(r) \leq \varphi^{\prime}(r) \quad \text { and } \quad \varphi(r) \leq c \mu(r) \quad \text { for } \quad r>0 .
$$

Then problem (1.1) has a unique solution in the class of weak solutions satisfying

$$
b(u), b(v) \in L^{1}(0, T ; \mathcal{W}),
$$

with $\mathcal{W}$ given by (3.1).

Proof. Consider, as in proof of Theorem 2.1, the sequence of regularized problems $(1.1)_{\varepsilon}$ in which we approximate solutions of the degenerate problem (1.1) by taking the perturbed auxiliary conditions given by (2.39), remaining the other conditions unchanged. We know from Theorem 2.2 that for each $\varepsilon>0$ problem $(1.1)_{\varepsilon}$ has, at least, a weak solution $\left(u_{\varepsilon}, v_{\varepsilon}, w_{\varepsilon}\right)$ with the additional regularity stated in the mentioned theorem and converging to a weak solution $(u, v, w)$ of (1.1) (Theorem 2.1). Moreover, there exist positive constants $\lambda$ and $c$, independent of $\varphi$ and $\varepsilon$, such that

$$
c \geq u_{\varepsilon}, v_{\varepsilon} \geq \varepsilon e^{-\lambda t} \quad \text { a.e. in } Q_{T}
$$

and

$$
\left\|\nabla w_{\varepsilon}\right\|_{L^{\infty}\left(Q_{T}\right)} \leq c .
$$

Suppose that $\left(u_{2}, v_{2}, w_{2}\right)$ is another weak solution of (1.1) and define $\left(U_{\varepsilon}, V_{\varepsilon}, W_{\varepsilon}\right):=$ $\left(u_{\varepsilon}-u_{2}, v_{\varepsilon}-v_{2}, w_{\varepsilon}-w_{2}\right)$ and $F_{\varepsilon}:=F\left(u_{\varepsilon}, v_{\varepsilon}\right)-F\left(u_{2}, v_{2}\right)$. Then $\left(U_{\varepsilon}, V_{\varepsilon}, W_{\varepsilon}\right)$ satisfy

$$
\left(3 . 3 4 \left\{\begin{array}{l}
U_{\varepsilon t}-\Delta\left(\varphi\left(u_{\varepsilon}\right)-\varphi\left(u_{2}\right)\right)+\operatorname{div}\left(b\left(u_{\varepsilon}\right) \nabla W_{\varepsilon}+\left(b\left(u_{\varepsilon}\right)-b\left(u_{2}\right)\right) \nabla w_{2}\right)=F_{\varepsilon}, \\
V_{\varepsilon t}-\Delta\left(\varphi\left(v_{\varepsilon}\right)-\varphi\left(v_{2}\right)\right)-\operatorname{div}\left(b\left(v_{\varepsilon}\right) \nabla W_{\varepsilon}+\left(b\left(v_{\varepsilon}\right)-b\left(v_{2}\right)\right) \nabla w_{2}\right)=F_{\varepsilon}, \\
-\Delta W_{\varepsilon}+U_{\varepsilon}-V_{\varepsilon}=0,
\end{array}\right.\right.
$$

in $Q_{T}$, and the auxiliary conditions

$$
(3.35) \begin{cases}\varphi\left(u_{D \varepsilon}\right)=\varphi\left(u_{D}\right)+\varphi\left(\varepsilon e^{-\lambda_{1} t}\right), \quad \varphi\left(v_{D \varepsilon}\right)=\varphi\left(v_{D}\right)+\varphi\left(\varepsilon e^{-\lambda_{1} t}\right) & \text { on } \Sigma_{D}, \\ \varphi\left(u_{D 2}\right)=\varphi\left(u_{D}\right), \varphi\left(v_{D 2}\right)=\varphi\left(v_{D}\right), \quad W_{D \varepsilon}=0 & \text { on } \Sigma_{D}, \\ \nabla \varphi\left(u_{N \varepsilon}\right) \cdot \nu=\nabla \varphi\left(v_{N \varepsilon}\right) \cdot \nu=\nabla W_{\varepsilon} \cdot \nu=0 & \text { on } \Sigma_{N}, \\ \nabla \varphi\left(u_{2}\right) \cdot \nu=\nabla \varphi\left(v_{2}\right) \cdot \nu=0 & \text { on } \Sigma_{N}, \\ U_{\varepsilon}(\cdot, 0)=V_{\varepsilon}(\cdot, 0)=\varepsilon & \text { in } \Omega .\end{cases}
$$

Taking smooth test functions $\psi, \xi, \eta$ with homogeneous mixed boundary conditions for problem (3.34)-(3.35) we get

$$
\begin{aligned}
\int_{\Omega} \psi(T) U_{\varepsilon}(T)+\xi(T) V_{\varepsilon}(T)= & \varepsilon \int_{\Omega} \psi(0)+\xi(0)-\int_{\Sigma_{D}} \varphi\left(\varepsilon e^{-\lambda_{1} t}\right)(\nabla \psi+\nabla \xi) \cdot \nu \\
& +\int_{Q_{T}} U_{\varepsilon}\left(\psi_{t}+A_{u}^{\varepsilon} \Delta \psi+\mathbf{B}_{u}^{\varepsilon} \cdot \nabla \psi+\eta+F_{u}^{\varepsilon}(\psi+\xi)\right) \\
& +\int_{Q_{T}} V_{\varepsilon}\left(\xi_{t}+A_{v}^{\varepsilon} \Delta \xi-\mathbf{B}_{v}^{\varepsilon} \nabla \xi-\eta+F_{v}^{\varepsilon}(\psi+\xi)\right) \\
& -\int_{Q_{T}} W_{\varepsilon} \operatorname{div}\left(b\left(u_{\varepsilon}\right) \nabla \psi-b\left(v_{\varepsilon}\right) \nabla \xi+\nabla \eta\right),
\end{aligned}
$$

with $A_{z}^{\varepsilon}:=\int_{0}^{1} \varphi^{\prime}\left(s z_{\varepsilon}+(1-s) z_{2}\right) d s, \mathbf{B}_{z}^{\varepsilon}:=\nabla w_{2} \int_{0}^{1} b^{\prime}\left(s z_{\varepsilon}+(1-s) z_{2}\right) d s$ for $z=u, v$ and $F_{u}^{\varepsilon}:=\int_{0}^{1} \frac{\partial F}{\partial u}\left(s u_{\varepsilon}+(1-s) u_{2}, v_{\varepsilon}\right) d s$ with a similar definition for $F_{v}^{\varepsilon}$, where here 
and in the sequel we denote by $z$ any of the functions $u$ or $v$. Due to $b \in \mathcal{C}^{1}([0, \infty))$, $F$ Lipschitz continuous, (3.32) and (3.33) we have

$$
\left\|\mathbf{B}_{z}^{\varepsilon}\right\|_{L^{\infty}\left(Q_{T}\right)},\left\|F_{z}^{\varepsilon}\right\|_{L^{\infty}\left(Q_{T}\right)} \leq k_{0},
$$

with $k_{0}$ independent of $\varepsilon$. In addition, using (3.31) we deduce the existence of a constant

$$
k(\varepsilon):=\varepsilon^{-1} e^{\lambda T} \mu\left(\varepsilon e^{-\lambda T}\right)
$$

such that

$$
0<k(\varepsilon) \leq A_{z}^{\varepsilon} \leq k_{0} \quad \text { in } Q_{T} .
$$

We consider sequences of $\mathcal{C}^{\infty}\left(Q_{T}\right)$ functions such that

$$
A_{z}^{\varepsilon, n} \rightarrow A_{z}^{\varepsilon}, \quad \mathbf{B}_{z}^{\varepsilon, n} \rightarrow \mathbf{B}_{z}^{\varepsilon}, \quad F_{z}^{\varepsilon, n} \rightarrow F_{z}^{\varepsilon}, \quad \text { y } \quad b_{z}^{n} \rightarrow b\left(z_{\varepsilon}\right),
$$

strongly in $L^{2}\left(Q_{T}\right)$ when $n \rightarrow \infty$. We choose $A_{z}^{\varepsilon, n}$ monotone decreasing on $n$ and $\mathbf{B}_{z}^{\varepsilon, n}$, $F_{z}^{\varepsilon, n}$ and $b_{z}^{n}$ monotone increasing on $n$. Because of $(3.39),(3.37)$ and the $L^{\infty}\left(Q_{T}\right)$ regularity of solutions of (1.1) we deduce

$\left(3.40 \nless k(\varepsilon) \leq A_{z}^{\varepsilon, n} \leq k_{0}, \quad\right.$ and $\quad\left\|\mathbf{B}_{z}^{\varepsilon, n}\right\|_{L^{\infty}\left(Q_{T}\right)},\left\|F_{z}^{\varepsilon, n}\right\|_{L^{\infty}\left(Q_{T}\right)},\left\|b_{z}^{\varepsilon, n}\right\|_{L^{\infty}\left(Q_{T}\right)} \leq k_{0}$ in $Q_{T}$. We rewrite identity (3.36) as

$$
\begin{aligned}
\int_{\Omega} \psi(T) U_{\varepsilon}(T)+\xi(T) V_{\varepsilon}(T) & =\varepsilon \int_{\Omega} \psi(0)+\xi(0)-\int_{\Sigma_{D}} \varphi\left(\varepsilon e^{-\lambda_{1} t}\right)(\nabla \psi+\nabla \xi) \cdot \nu \\
& +\int_{Q_{T}} U_{\varepsilon}\left[\left(A_{u}^{\varepsilon}-A_{u}^{\varepsilon, n}\right) \Delta \psi+\left(\mathbf{B}_{u}^{\varepsilon}-\mathbf{B}_{u}^{\varepsilon, n}\right) \cdot \nabla \psi\right] \\
& +\int_{Q_{T}} V_{\varepsilon}\left[\left(A_{v}^{\varepsilon}-A_{v}^{\varepsilon, n}\right) \Delta \xi-\left(\mathbf{B}_{v}^{\varepsilon}-\mathbf{B}_{v}^{\varepsilon, n}\right) \nabla \xi\right] \\
& +\int_{Q_{T}} U_{\varepsilon}\left(F_{u}^{\varepsilon}-F_{u}^{\varepsilon, n}\right)(\psi+\xi)+V_{\varepsilon}\left(F_{v}^{\varepsilon}-F_{v}^{\varepsilon, n}\right)(\psi+\xi) \\
& +\int_{Q_{T}} \nabla W_{\varepsilon} \cdot\left(\left(b\left(u_{\varepsilon}\right)-b_{u}^{n}\right) \nabla \psi-\left(b\left(v_{\varepsilon}\right)-b_{v}^{n}\right) \nabla \xi\right) \\
& -\int_{Q_{T}} W_{\varepsilon} d i v\left(b_{u}^{n} \nabla \psi-b_{v}^{n} \nabla \xi+\nabla \eta\right) \\
& +\int_{Q_{T}} U_{\varepsilon}\left(\psi_{t}+A_{u}^{\varepsilon, n} \Delta \psi+\mathbf{B}_{u}^{\varepsilon, n} \cdot \nabla \psi+\eta+F_{u}^{\varepsilon, n}(\psi+\xi)\right) \\
& +\int_{Q_{T}} V_{\varepsilon}\left(\xi_{t}+A_{v}^{\varepsilon, n} \Delta \xi-\mathbf{B}_{v}^{\varepsilon, n} \cdot \nabla \xi-\eta+F_{v}^{\varepsilon, n}(\psi+\xi)\right) \\
= & I_{1}+\cdots+I_{9},
\end{aligned}
$$

and set the following problem to choose the test functions:

$$
\begin{cases}\psi_{t}+A_{u}^{\varepsilon, n} \Delta \psi+\mathbf{B}_{u}^{\varepsilon, n} \cdot \nabla \psi+\eta+F_{u}^{\varepsilon, n}(\psi+\xi)=0 & \text { in } Q_{T}, \\ \xi_{t}+A_{v}^{\varepsilon, n} \Delta \xi-\mathbf{B}_{v}^{\varepsilon, n} \cdot \nabla \xi-\eta+F_{v}^{\varepsilon, n}(\psi+\xi)=0 & \text { in } Q_{T}, \\ \Delta \eta+\operatorname{div}\left(b_{u}^{n} \nabla \psi-b_{v}^{n} \nabla \xi\right)=0 & \text { in } Q_{T}, \\ \psi=\xi=\eta=0 & \text { on } \Sigma_{D}, \\ \nabla \psi \cdot \nu=\nabla \xi \cdot \nu=\nabla \eta \cdot \nu=0 & \text { on } \Sigma_{N}, \\ \psi(\cdot, T)=\chi_{\delta}^{u}, \quad \xi(\cdot, T)=\chi_{\delta}^{v} & \text { in } \Omega,\end{cases}
$$


with $\chi_{\delta}^{z} \in \mathcal{C}_{0}^{\infty}(\Omega)$ satisfying $\operatorname{dist}\left(\Sigma_{D}, \operatorname{supp}\left(\chi_{\delta}^{z}\right)\right) \geq \delta$ and $\chi_{\delta}^{z}$ uniformly bounded in $L^{1}(\Omega)$ for all $\delta>0$.

LEMma 3.5. Problem (3.42) has a unique solution with the regularity of test functions of (1.1), see (2.4) and (2.5). Moreover,

$$
\begin{gathered}
\psi, \xi \in H^{1}\left(0, T ; L^{2}(\Omega)\right) \cap L^{\infty}\left(0, T ; H^{1}(\Omega)\right) \cap L^{2}\left(0, T ; H^{2}(\Omega)\right), \\
\eta \in H^{1}\left(0, T ; L^{2}(\Omega)\right) \cap L^{\infty}\left(0, T ; H^{1}(\Omega)\right),
\end{gathered}
$$

and their norms in these spaces are uniformily bounded with respect to $n$. Finally, there exists a positive constant $C(T)$ independent of $\varepsilon$ and $\delta$ such that

$$
\|\psi\|_{L^{\infty}\left(Q_{T}\right)},\|\xi\|_{L^{\infty}\left(Q_{T}\right)} \leq C(T) .
$$

Continuation of proof of Theorem 3.4. With the test functions of Lemma 3.5 we have $I_{7}=I_{8}=I_{9}=0$ in (3.41). Using in the resulting identity the uniform estimates with respect to $n$ provided by Lemma 3.5 we deduce that $I_{3}, I_{4}, I_{5}$ and $I_{6}$ tend to zero when $n \rightarrow \infty$. Therefore, identity (3.41) is reduced to

$(3.45) \int_{\Omega} \chi_{\delta}^{u} U_{\varepsilon}(T)+\chi_{\delta}^{v} V_{\varepsilon}(T)=\varepsilon \int_{\Omega}(\psi(0)+\xi(0))-\int_{\Sigma_{D}} \varphi\left(\varepsilon e^{-\lambda_{1} t}\right)(\nabla \psi+\nabla \xi) \cdot \nu$.

The treatment of the boundary integral deserves the following

Lemma 3.6. Let $A_{\varepsilon}, \mathbf{B}_{\varepsilon}, g_{\varepsilon} \in L^{\infty}\left(Q_{T}\right)$ with

$$
k(\varepsilon)<A_{\varepsilon},
$$

where $k(\varepsilon)$ is given by (3.38). Consider the problem

$$
\begin{cases}\psi_{t}+A_{\varepsilon} \Delta \psi+\mathbf{B}_{\varepsilon} \cdot \nabla \psi+g_{\varepsilon}=0 & \text { in } Q_{T}, \\ \psi=0 & \text { on } \Sigma_{D}, \\ \nabla \psi \cdot \nu=0 & \text { on } \Sigma_{N}, \\ \psi(\cdot, T)=\chi_{\delta} & \text { in } \Omega,\end{cases}
$$

with $\delta>0$. Then, there exist $a \delta(\varepsilon)>0$ and a positive constant $c$, independent of $\varepsilon$, such that if $\delta<\delta(\varepsilon)$ then

$$
-\nabla \psi \cdot \nu \leq c \frac{\left\|\mathbf{B}_{\varepsilon}\right\|_{L^{\infty}\left(Q_{T}\right)}\|\psi\|_{L^{\infty}\left(Q_{T}\right)}}{k(\varepsilon)} \quad \text { a.e. in } \quad \Sigma_{D}
$$

End of proof of Theorem 3.4. Now we are in a position to pass to the limits $\varepsilon, \delta \rightarrow$ 0 . First note that Lemma 3.5 ensures the existence of uniform in $\varepsilon, \delta$ estimates of $\|\psi\|_{L^{\infty}\left(Q_{T}\right)},\|\xi\|_{L^{\infty}\left(Q_{T}\right)}$. Therefore

$$
\varepsilon \int_{\Omega}(\psi(0)+\xi(0)) \rightarrow 0 \quad \text { as } \quad \varepsilon, \delta \rightarrow 0 .
$$

Applying Lemma 3.6 to the two first equations of problem (3.42) and considering the uniform bounds in $\varepsilon$ of $\left\|\mathbf{B}_{u}^{\varepsilon}\right\|_{L^{\infty}\left(Q_{T}\right)}$, see (3.37), and in $\varepsilon, \delta$ of $\|\psi\|_{L^{\infty}\left(Q_{T}\right)}$, see Lemma 3.5 , we obtain

$$
-\int_{\Sigma_{D}} \varphi\left(\varepsilon e^{-\lambda_{1} t}\right) \nabla \psi \cdot \nu \leq c \frac{\varphi(\varepsilon)}{k(\varepsilon)}
$$


where we used that $\varphi$ is non-negative and increasing. Using (3.31) and (3.38) we obtain $\varphi(\varepsilon) \leq c \varepsilon k(\varepsilon)$ and from (3.49) we deduce

$$
-\int_{\Sigma_{D}} \varphi\left(\varepsilon e^{-\lambda_{1} t}\right) \nabla \psi \cdot \nu \leq 0 \quad \text { as } \quad \varepsilon, \delta \rightarrow 0 .
$$

A similar argument may be applied to the term involving $\xi$. To finish, we choose function $\chi_{\delta}^{u}$ as follows: set $\Omega_{\delta}:=\left\{x \in \Omega: \operatorname{dist}\left[\partial \Omega, \operatorname{supp}\left(U_{\varepsilon}(x, T)\right)\right]>\delta\right\}$ and define

$$
\chi_{\delta}^{u}(x, t):= \begin{cases}\operatorname{sign}\left\{u(x, t)-u_{2}(x, T)\right\} & \text { if } x \in \Omega_{\delta} \\ 0 & \text { if } x \in \Omega \backslash \bar{\Omega}_{\delta} .\end{cases}
$$

Then

$$
\int_{\Omega} \chi_{\delta}^{u} U_{\varepsilon}(T) \rightarrow \int_{\Omega}\left|u(T)-u_{2}(T)\right| \quad \text { as } \quad \varepsilon, \delta \rightarrow 0,
$$

and a similar property holds for the term involving $V_{\varepsilon}$. Gathering (3.48)-(3.51) we deduce from (3.45)

$$
\int_{\Omega}\left|u(T)-u_{2}(T)\right|+\left|v(T)-v_{2}(T)\right| \leq 0,
$$

and the assertion follows.

Proof of Lemma 3.5. The proof follows the same scheme than proof of Lemma 3.2. Therefore, we shall only show how to obtain property (3.44). Set

$K:=\left\{h \in L^{2}\left(0, T^{*} ; \mathcal{V}\right) \cap L^{1}\left(0, T^{*} ; L^{\infty}(\Omega)\right):\|h\|_{L^{2}\left(0, T^{*} ; \mathcal{V}\right)}+\|h\|_{L^{1}\left(0, T^{*} ; L^{\infty}(\Omega)\right)}<R\right\}$, and define $Q: K \rightarrow L^{2}\left(0, T^{*} ; \mathcal{V}\right) \cap L^{1}\left(0, T^{*} ; L^{\infty}(\Omega)\right)$ by $Q(\hat{\eta})=\eta$, being $\eta$ the solution of

$$
\begin{cases}\Delta \eta+\operatorname{div}\left(b_{u}^{n} \nabla \hat{\psi}-b_{v}^{n} \nabla \hat{\xi}\right)=0 & \text { in } Q_{T}, \\ \eta=0 & \text { on } \Sigma_{D} \\ \nabla \eta \cdot \nu=0 & \text { on } \Sigma_{N}\end{cases}
$$

with $(\hat{\psi}, \hat{\xi})$ solution of

$$
\begin{cases}\hat{\psi}_{t}+A_{u}^{\varepsilon, n} \Delta \hat{\psi}+\mathbf{B}_{u}^{\varepsilon, n} \cdot \nabla \hat{\psi}+\hat{\eta}+F_{u}^{\varepsilon, n}(\hat{\psi}+\hat{\xi})=0 & \text { in } Q_{T}, \\ \hat{\xi}_{t}+A_{v}^{\varepsilon, n} \Delta \hat{\xi}-\mathbf{B}_{v}^{\varepsilon, n} \cdot \nabla \hat{\xi}-\hat{\eta}+F_{v}^{\varepsilon, n}(\hat{\psi}+\hat{\xi})=0 & \text { in } Q_{T}, \\ \hat{\psi}=\hat{\xi}=0 & \text { on } \Sigma_{D}, \\ \nabla \hat{\psi} \cdot \nu=\nabla \hat{\xi} \cdot \nu=0 & \text { on } \Sigma_{N}, \\ \hat{\psi}(\cdot, T)=\chi_{\delta}^{u}, \quad \hat{\xi}(\cdot, T)=\chi_{\delta}^{v} & \text { in } \Omega .\end{cases}
$$

By Alexandrov's maximum principle, see [27], the solution of (3.53) satisfy

$$
\|\hat{\psi}\|_{L^{\infty}\left(Q_{T^{*}}\right)}+\|\hat{\xi}\|_{L^{\infty}\left(Q_{T^{*}}\right)} \leq e^{c T^{*}}\|\hat{\eta}\|_{L^{1}\left(0, T^{*} ; L^{\infty}(\Omega)\right)},
$$

with $c$ depending on a bound of $\left\|F_{z}^{\varepsilon, n}\right\|_{L^{\infty}}$ which we know independent of $\varepsilon, n$, see (3.40). Now define

$$
\theta:=\eta+b_{u}^{n} \hat{\psi}-b_{v}^{n} \hat{\xi}
$$


From (3.52) we have that $\theta$ satisfies

$$
\begin{cases}\Delta \theta=\operatorname{div}\left(\hat{\psi} \nabla b_{u}^{n}-\hat{\xi} \nabla b_{v}^{n}\right) & \text { in } Q_{T} \\ \theta=0 & \text { on } \Sigma_{D} \\ \nabla \theta \cdot \nu=0 & \text { on } \Sigma_{N}\end{cases}
$$

By well known results, see [40], we have

$$
\|\theta\|_{L^{\infty}(\Omega)} \leq c\left(\left\|\hat{\xi} \nabla b_{v}^{n}\right\|_{L^{p}(\Omega)}+\left\|\hat{\psi} \nabla b_{u}^{n}\right\|_{L^{p}(\Omega)}\right),
$$

for $p$ given in (3.1). Then

$$
\|\theta\|_{L^{\infty}(\Omega)} \leq c\left(\|\hat{\xi}\|_{L^{\infty}(\Omega)}\left\|\nabla b_{v}^{n}\right\|_{L^{p}(\Omega)}+\|\hat{\psi}\|_{L^{\infty}(\Omega)}\left\|\nabla b_{u}^{n}\right\|_{L^{p}(\Omega)}\right) .
$$

Recalling the definition of $\theta$ and using the assumption $b(u), b(v) \in L^{1}(0, T ; \mathcal{W})$ we obtain

$$
\|\eta\|_{L^{1}\left(0, T^{*} ; L^{\infty}(\Omega)\right)} \leq c\left(T^{*}\right)\left(\|\hat{\psi}\|_{L^{\infty}\left(Q_{T^{*}}\right)}+\|\hat{\xi}\|_{L^{\infty}\left(Q_{T^{*}}\right)}\right)
$$

with $c(t)$ depending only on $\|\nabla b(z)\|_{L^{1}\left(0, t ; L^{p}(\Omega)\right)}$ and $\|b(z)\|_{L^{\infty}\left(Q_{t}\right)}, z=u, v$. Gathering (3.54) and (3.56) we find

$$
\|\eta\|_{L^{1}\left(0, T^{*} ; L^{\infty}(\Omega)\right)} \leq c\left(T^{*}\right) e^{c T^{*}}\|\hat{\eta}\|_{L^{1}\left(0, T^{*} ; L^{\infty}(\Omega)\right)}<c\left(T^{*}\right) e^{c T^{*}} R,
$$

and to obtain $Q(K) \subset K$ we only have to choose $T^{*}$ small enough. The rest of the proof of existence of a fixed point is similar as that in Lemma 3.2. Finally, notice that a continuation argument allows to extend the solution to any $T>0$. Indeed, estimates (3.54) and (3.56) do not blow up for any finite $T^{*}$.

Proof of Lemma 3.6. Since $\partial \Omega$ is regular, $\Omega$ satisfies the exterior sphere condition, i.e., for all $x_{0} \in \partial \Omega$ there exists $R_{1}>0$ and $x_{1} \in \mathbb{R}^{N} \backslash \bar{\Omega}$ such that

$$
B\left(x_{1}, R_{1}\right) \cap \bar{\Omega}=\left\{x_{0}\right\},
$$

where $B\left(x_{1}, R_{1}\right):=\left\{x \in \mathbb{R}^{N}:\left|x-x_{1}\right|<R_{1}\right\}$. Let us fix $x_{0} \in \operatorname{Interior}\left(\Gamma_{D}\right)$. This set is non empty because, by hypothesis, there exists an open set $\tilde{B}$ such that $\tilde{B} \subset \Gamma_{D}$. Therefore, there exists a small enough $\delta>0$ such that, by defining $R_{2}:=\delta+R_{1}$, we have $B\left(x_{1}, R_{2}\right) \cap \partial \Omega \subset \Gamma_{D}$. Moreover, since $\operatorname{dist}\left(\partial \Omega, \operatorname{supp}\left(\chi_{\delta}\right)\right) \geq \delta$, we also have $\chi_{\delta} \equiv 0 \quad$ in $\quad \omega:=\Omega \cap B\left(x_{1}, R_{2}\right)$. We shall use the notation $k_{0}(\varepsilon):=\|g\|_{L^{\infty}\left(Q_{T}\right)}$, $k_{1}(\varepsilon):=\left(\frac{N-1}{R_{1}}+1\right)\|\mathbf{B}\|_{L^{\infty}\left(Q_{T}\right)}$ and $k_{2}(\varepsilon):=\|\psi\|_{L^{\infty}\left(Q_{T}\right)}$. We define

$$
\mathcal{L}(\psi):=\psi_{t}+A_{\varepsilon} \Delta \psi+\mathbf{B} \cdot \nabla \psi \quad \text { and } \quad w(x, t):=\psi(x, t)+\sigma(r),
$$

with $(x, t) \in \omega \times(0, t)$ and $r:=\left|x-x_{0}\right|$. Function $\sigma \in C^{2}\left(\left[R_{1}, R_{2}\right]\right)$ will be chosen such that $\sigma^{\prime \prime}(r) \geq 0, \sigma^{\prime}(r) \leq 0$ and the maximum of $w$ in $\bar{\omega} \times[0, T]$ is attained in $\left\{x_{0}\right\} \times[0, T]$. Assuming these properties we get, due to (3.46)

$$
\mathcal{L}(w)=-g+A_{\varepsilon} \Delta \sigma+\mathbf{B} \cdot \nabla \sigma \geq k(\varepsilon) \sigma^{\prime \prime}(r)+k_{1}(\varepsilon) \sigma^{\prime}(r)-k_{0}(\varepsilon)
$$


Choosing $\sigma(r):=\frac{k_{0}(\varepsilon)}{k_{1}(\varepsilon)} r+C_{2} e^{-\frac{k_{1}(\varepsilon)}{k(\varepsilon)} r}$, with $C_{2}$ an arbitrary constant, we obtain

$$
\begin{gathered}
k(\varepsilon) \sigma^{\prime \prime}(r)+k_{1}(\varepsilon) \sigma^{\prime}(r)-k_{0}(\varepsilon)=0, \quad \sigma^{\prime \prime}(r) \geq 0 \quad \text { and } \\
\quad \text { if } \quad C_{2} \geq k(\varepsilon) \frac{k_{0}(\varepsilon)}{k_{1}^{2}(\varepsilon)} e^{\frac{k_{1}(\varepsilon)}{k(\varepsilon)} R_{2}} \quad \text { then } \quad \sigma^{\prime}(r) \leq 0 .
\end{gathered}
$$

Taking $C_{2}$ with this restriction we have that $\mathcal{L}(w) \geq 0$ in $\bar{\omega} \times[0, T]$ and therefore, by the maximum principle, we deduce that $w$ attains its maximum on the parabolic boundary of $\omega \times[0, T]$. On this boundary the values of $w$ may be estimated as follows:

$$
\begin{cases}w(x, t)=\sigma(r) \leq \sigma\left(R_{1}\right) & \text { on }\left(\Gamma_{D} \cap \partial \omega\right) \times[0, T], \\ w(x, t)=\psi(x, t)+\sigma(r) \leq k_{2}(\varepsilon)+\sigma\left(R_{2}\right) & \text { on }\left(\partial B\left(x_{1}, R_{2}\right) \cap \partial \omega\right) \times[0, T] \\ w\left(x_{0}, t\right)=\sigma\left(R_{1}\right) & \text { on }[0, T] \\ w(x, T)=\sigma(r)+\chi_{\delta}(x) \leq \sigma\left(R_{1}\right) & \text { in } \omega,\end{cases}
$$

where we have used that $\chi_{\delta} \equiv 0$ in $\omega$. It is a straightforward computation to see that, by making $\delta$ small enough, we can choose $C_{2}$ such that $(3.57)$ and $\sigma\left(R_{1}\right)=$ $k_{2}(\varepsilon)+\sigma\left(R_{2}\right)$ hold. As a consequence we obtain that $\nabla w\left(x_{0}, t\right) \cdot \nu \geq 0$ and by the definition of $w$ and taking $\delta$ suitably we obtain

$$
-\nabla \psi\left(x_{0}, t\right) \cdot \nu \leq c \frac{k_{1}(\varepsilon) k_{2}(\varepsilon)}{k(\varepsilon)} \quad \text { in }[0, T]
$$

\section{REFERENCES}

[1] Alt, H.W. And Luckhaus, S., Quasilinear Elliptic-Parabolic Differential Equations, Math. Z., $183,311-341,1983$.

[2] Antontsev, S.N., Díaz, J.I. And Domansky, A.V., Continuous dependence and stabilization of solutions of the degenerate system in two-phase filtration, Dinamika sploshnoi sredy, No. 107, 11-25, 1993.

[3] Antontsev, S.N., Kazhikov, A.V. and Monkhov, V.N., Boundary Value Problems in Mechanics of Nonhomogeneous Fluids, North-Holland, Amsterdam, 1990.

[4] Arino, O., Gauthier, S. and Penot, J.P., A fixed point theorem for sequentially continuous mappings with application to ordinary differential equations, Funkcial Ekvac., 27, 273-279, 1987.

[5] Benilan, P., Equations d'evolution dans un espace de Banach quelconque et applications, Thése, Orsay, 1972.

[6] Benilan, P. and Gariepy, R., Strong solutions in $L^{1}$ of degenerate parabolic equations. $J$. Diff. Eq., 119, 473-502, 1995.

[7] Bertsch, M. and Hilhorst, D., A density dependent diffusion equation in Population Dynamics: stabilization to equilibrium, SIAM J. Math. Anal., 17, 4, 863-883, 1986.

[8] BrÉzis, H., Opérateurs Maximaux Monotones et Semigroupes de Contractions dans les Espaces de Hilbert, North Holland, Amsterdam, 1973.

[9] Carrillo, J., On the uniqueness of the solution of the evolution dam problem, Nonlinear Analysis, Theory, Methods and Applications, 22, 5, 573-607, 1994.

[10] Courant, R. and Friedrichs, K.O., Supersonic Flow and Shock Waves, Interscience, 1967.

[11] Díaz, J.I., Galiano, G. And Jüngel, A., Space localization and uniqueness of solutions of a quasilinear parabolic system arising in semiconductor theory, Comptes Rendus Academie Sciences, t. 325, Serie I, 267-272, 1997.

[12] Díaz, J.I., Galiano, G. and Jüngel, A., On a quasilinear degenerate system arising in semiconductors theory. Part II: Localization of vacuum solutions, to appear in Nonlinear Analysis, Serie B, 1997.

[13] Díaz, J.I. And Kersner, R., On a nonlinear degenerate parabolic equation in infiltration or evaporation through a porous medium, J. Differential Eq., 69, 368-403, 1987.

[14] Díaz, J.I. and Padial, J.F., Uniqueness and existence of solutions in $B V_{t}(Q)$ space to a doubly nonlinear parabolic problem, Plublicacions Matematiques, 40, 527-560, 1996. 
[15] Díaz and J.I., Vrabie, I.I., Existence for reaction diffusion systems: A compactness approach, J. Math. Anal. Appl., 188, 521-540, 1994.

[16] Díaz and J.I., Vrabie, I.I., Compactness of the Green Operator of nonlinear diffusion equations: applications to Boussinesq type systems in Fluid Dynamics, Topological Methods in Nonlinear Analysis, 4, 399-416, 1994.

[17] Dubinskil, J.A., Weak convergence in nonlinear elliptic and parabolic equations, A.M.S. Transl., 67, 226-258, 1968.

[18] Gagneux, G. and Madaune-Tort, M., Unicité des solutions faibles d'équations de diffusionconvection, Comptes Rendus Academie Sciences, t. 318, Série I, 919-924, 1994.

[19] Gagneux, G. and Madaune-Tort, M., Analyse mathematique de modeles non lineaires de l'ingenierie petroliere, Springer, Paris, 1996.

[20] Galiano, G., Sobre algunos problemas de la Mecánica de Medios Continuos en los que se originan Fronteras Libres, Thesis, Universidad Complutense de Madrid, 1997.

[21] Gilding, B.H., Improved theory for a nonlinear degenerate parabolic equation, Ann. Sc. Norm. Sup. Pisa, Cl. Sci. IV, 16, 165-224, 1989.

[22] JüNGEL, A., On the existence and uniqueness of transient solutions of a degenerate nonlinear drift-diffusion model for semiconductors, Math. Models Meth. Appl. Sci., 4:677-703, 1994.

[23] JÜNGEL, A., Qualitative behavior of solutions of a degenerate nonlinear drift-diffusion model for semiconductors, Math. Models Meth. Appl. Sci., 5:497-518, 1995.

[24] Jüngel, A., Asymptotic analysis of a semiconductor model based on Fermi-Dirac statistics, Math. Meth. Appl. Sci., 19:401-424, 1996.

[25] JüNGEL, A., A nonlinear drift-diffusion system with electric convection arising in semiconductor and electrophoretic modeling, Math. Nachr. 185, 85-110, 1997.

[26] Kalashnikov, A.S., The Cauchy problem in a class of growing functions for equations of unsteady filtration type, Vestnik Moskov Univ. Ser. VI Mat. Mech., 6, 17-27, 1963.

[27] Krylov, N.V., Nonlinear elliptic and parabolic equations of the second order, D. Reidel Publishing Company, Dordrecht, 1987.

[28] Kruzhkov, S.N., First order quasilinear equations in several independent variables, Math. USSR Sbornik, 10, No 2, 217-243, 1970.

[29] Kruzhkov, S.N. AND SukorJanski, S.M., Boundary value problems for systems of equations of two phase porous flow type: statement of the problems, questions of solvability, justification of approximate methods, Math USSR Sbornik, 33, No 1, 62-80, 1977.

[30] Ladyzenskaya, O.A., Solonnikov, V.A. and Ural'ceva, N.N., Quasilinear Equations of Parabolic Type, Translations of Mathematical Monographs, 23, American Mathematical Society, Providence, 1968 .

[31] Lions, J.L., Quelques méthodes de résolution des problèmes aux limites non linéaires, Dunod, Gauthiers-Villars, Paris 1969.

[32] Markovich, P.A., The stationary semiconductor device equations, Springer-Verlag, Wien, 1986.

[33] Markovich, P.A., Ringhofer, C.A. and Schmeiser, C., Semiconductor Equations, SpringerVerlag, Wien, 1990

[34] Mock, M.S. On equations describing steady state carrier distributions in a semiconductor device, Comm. Pure Appl. Math., 25, 781-792, 1972.

[35] Отто, F., $L^{1}$-contraction and uniqueness for quasilinear elliptic-parabolic equations. J. Diff. Equations, 131, 20-38, 1996.

[36] PADiAL, J.F., A comparison principle for weak solutions of some nonlinear parabolic systems, preprint, 1997.

[37] Rulla, J., Weak solutions to Stefan Problems with prescribed convection, SIAM J. Math. Anal., 18, 6, 1784-1800, 1987.

[38] Shalimova, K.V., Física de los Semiconductores, Mir, Moscow, 1973.

[39] Simon, J., Compact sets in the space $L^{p}(0, T ; B)$, Ann. Math. Pura et Appl., 146, 65-96, 1987.

[40] Stampacchia, G., Equations elliptiques a données discontinues, Seminaire Schwartz, 1960-61: Equations aux dérivées partialles et interpolation, 4.01-4.16.

[41] Troianiello, G.M., Elliptic differential equations and obstacle problems, Plenum Press, New York, 1987. 